\title{
Structural Evaluation of Protein/Metal Complexes via Native Electrospray Ultraviolet Photodissociation Mass Spectrometry
}

Christopher M. Crittenden ${ }^{1}$, Elisa T. Novelli ${ }^{1}$, M. Rachel Mehaffey ${ }^{1}$, Gulan N. Xu ${ }^{1}$, David H. Giles ${ }^{2}$, Whitney A. Fies ${ }^{1}$, Kevin N. Dalby ${ }^{2,3}$, Lauren J. Webb $b^{1,4,5}$, Jennifer S. Brodbelt ${ }^{* 1}$

${ }^{1}$ Department of Chemistry, University of Texas, Austin, Texas 78712, United States

${ }^{2}$ Division of Chemical Biology and Medicinal Chemistry, University of Texas, Austin, Texas 78712, United States

${ }^{3}$ Graduate Program in Cell and Molecular Biology, University of Texas, Austin, Texas 78712, United States

${ }^{4}$ Institute for Cell and Molecular Biology, University of Texas, Austin, Texas 78712, United States

${ }^{5}$ Texas Materials Institute, University of Texas, Austin, Texas 78712, United States

*Phone: (512) 471-0028, E-mail: brodbelt@cm.utexas.edu

\section{Supporting Information}

\section{Preparation of Proteins}

Staphylococcal Nuclease

A pET-42a+ plasmid containing the gene for Staphylococcal nuclease (Staphylococcus aureus) $\Delta+$ PHS I92C and a kanamycin resistance marker was a generous gift of Dr. Bertrand GarciaMoreno E. (The $\Delta+$ PHS variant denotes a deletion of residues $44-49$, and the mutations P117G, H124L, S128A, G50F, and V51N, as previously described) [1]. The cysteine at position 92 was reverted to the native isoleucine using the Quik-Change Lightning site-directed mutagenesis kit (Agilent) to generate the "wild-type" $\Delta+$ PHS form of Staphylococcal nuclease, hereafter referred to as SNase. The mutated plasmid was transformed into BL21-DE3 cells (New England BioLabs) and grown in LB-Broth containing kanamycin at 37 degrees $\mathrm{C}$ to an $\mathrm{OD}_{600} \sim 0.4-0.6$. Cells were induced with $1 \mathrm{mM}$ isopropyl $\beta$-D-1-thiogalactopyranoside (IPTG) for 4-6 hours, collected by centrifugation, flash frozen in liquid nitrogen, and stored at $-80^{\circ} \mathrm{C}$ for further use. Protein was purified from inclusion bodies based on a strategy described previously [2-4]. Briefly, cells were lysed by re-suspending in Buffer A containing $6 \mathrm{M}$ urea, $25 \mathrm{mM}$ Tris, and $2.5 \mathrm{mM}$ EDTA, pH 8. Cell debris (including inclusion bodies) was separated by centrifugation, and protein was freed from inclusion bodies by re-suspending in Buffer B (same as buffer A with the addition of 400 $\mathrm{mM} \mathrm{NaCl}$ ). Unfolded protein was collected and purified by two successive ethanol precipitation steps followed by further purification with cation exchange chromatography (SP sepharose, Fisher). The eluent containing SNase was precipitated a final time with ethanol, then re-suspending in fresh Buffer 2, and refolded by dialyzing overnight against $1 \mathrm{M} \mathrm{KCl}$, followed by dialyzing twice for 2 hours each against $\mathrm{ddH}_{2} \mathrm{O}$, and overnight with ddH2O. Protein refolding was confirmed by 
circular dichroism (CD) spectroscopy. Purified and refolded protein was diluted to $300 \mathrm{uM}$ with $\mathrm{ddH}_{2} \mathrm{O}$, separated into $1 \mathrm{ml}$ aliquots, and flash frozen for further use.

Azurin

The gene for azurin (Pseudomonas aeruginosa) was a gift from Dr. Judy Kim. Expression and purification was adapted from previously described protocols [5, 6]. Briefly, DNA was transformed into BL-21-DE3 cells and grown at $37^{\circ} \mathrm{C}$ in $\mathrm{LB}$ cultures containing ampicillin to OD 0.6. Expression was induced with $2 \mathrm{mM}$ IPTG for 4-6 hours at $37 \mathrm{deg}$ C. Cells were collected by centrifugation and washed with $10 \mathrm{mM}$ Tris $\mathrm{pH} 7.7$ before flash freezing and stored at -80 until purification. Cell pellets were resuspended in 50mL of $20 \mathrm{mM}$ potassium phosphate $\mathrm{pH} 7.2$ per 25 g pellet (approx 2 1L cultures). Cells were lysed by adding 200mg chicken egg lysozyme (Sigma) and 40-80uL of DNase 1 (Roche) and shaking at RT for 90 minutes. Lysate was separated from cell debris by centrifugation, and acidified with the addition of $2.5 \mathrm{ml}$ of $1 \mathrm{M}$ sodium acetate $\mathrm{pH} 4.2$ and sterile water to a final volume of $50 \mathrm{~mL}$. Precipitate was removed by centrifugation and supernatant containing azurin was saturated with copper by the addition of $250 \mathrm{mg}$ copper sulfate hexahydrate (sigma) and incubated for 1 week at $4{ }^{\circ} \mathrm{C}$, resulting in a dark blue copper-saturated, but impure, azurin solution. Azurin was further purified via fast protein liquid chromatography (FPLC) with a cation exchange column (Protein-PAK SP, Waters). Protein was loaded onto the column in $1 \mathrm{mM}$ sodium acetate $\mathrm{pH} 4.5$ and then washed with successive, high salt, then higher pH until eluted. Eluted azurin was purified by gel filtration chromatography on a Superdex 75 column (GE) in $1 \mathrm{mM}$ sodium acetate at low $\mathrm{pH}(4.5-5.5)$ and stored for further use.

\section{Calmodulin}

Calmodulin was expressed from the pET23 vector (a gift from the laboratory of M. Neal Waxham) in E. coli BL21 DE3, essentially as described by Gaertner et al [7]. Briefly, pelleted cells were

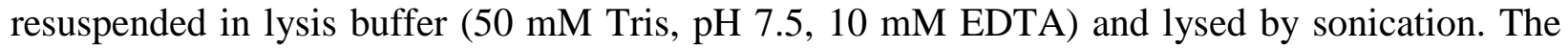
insoluble fraction was removed by centrifugation, followed by addition of ammonium sulfate to 2.5 $\mathrm{M}$ to the supernatant. Precipitant was removed by further centrifugation, followed by addition of saturating ammonium sulfate to the supernatant. This solution was centrifuged and the resulting pellet was resuspended in resuspension buffer $(200 \mathrm{mM}$ ammonium sulfate, $50 \mathrm{mM}$ Tris, $\mathrm{pH} 7.5$, $1 \mathrm{mM}$ EDTA, and a protease inhibitor cocktail consisting of $0.1 \% \beta$-mercaptoethanol, $1 \mathrm{mM}$ benzamidine, $0.1 \mathrm{mM}$ phenylmethylsulfonylfluoride (PMSF), and $0.1 \mathrm{mM}$ tosyl phenylalanyl chloromethyl ketone (TPCK). The resuspended pellet was subjected to FPLC over a phenyl 
Sepharose (PS) column. Flow through was collected and brought to $2.5 \mathrm{mM} \mathrm{CaCl}_{2}$ with stirring, followed by the gradual, stirred addition of ammonium sulfate to $200 \mathrm{mM}$. The PS column was washed with $6 \mathrm{M}$ Urea in $50 \mathrm{mM}$ Tris, $\mathrm{pH} 7.5$ to remove residual protein, and re-equilibrated in wash buffer (50 mM Tris, $\mathrm{pH}$ 7.5, $200 \mathrm{mM}$ ammonium sulfate, $2 \mathrm{mM} \mathrm{CaCl}_{2}$, plus protease inhibitor cocktail). The sample was run once more over the PS column, extensively washed, and eluted (50 $\mathrm{mM}$ Tris, $\mathrm{pH}$ 7.5, $1 \mathrm{M} \mathrm{NaCl}, 2.5 \mathrm{mM}$ EGTA, plus protease inhibitor cocktail). Calmodulincontaining fractions were collected, concentrated to $10 \mathrm{~mL}$, and subjected to gel filtration on a HiPrep 26/60 Sephacryl S-200 High Resolution FPLC column in 25 mM HEPES, pH 7.5. Calmodulin-containing fractions were concentrated to $227 \mu \mathrm{M}$ and stored at $-20{ }^{\circ} \mathrm{C}$ until further use.

\section{References}

1. García-Moreno, B.E., Dwyer, J.J., Gittis, A.G., Lattman, E.E., Spencer, D.S., Stites, W.E.: Experimental measurement of the effective dielectric in the hydrophobic core of a protein. Biophysical Chemistry. 64, 211224 (1997).

2. Karp, D.A., Gittis, A.G., Stahley, M.R., Fitch, C.A., Stites, W.E., García-Moreno E., B.: High Apparent Dielectric Constant Inside a Protein Reflects Structural Reorganization Coupled to the Ionization of an Internal Asp. Biophysical Journal. 92, 2041-2053 (2007).

3. Isom, D.G., Cannon, B.R., Castañeda, C.A., Robinson, A., E, B.G.-M.: High tolerance for ionizable residues in the hydrophobic interior of proteins. PNAS. 105, 17784-17788 (2008).

4. Shortle, D., Meeker, A.K.: Mutant forms of staphylococcal nuclease with altered patterns of guanidine hydrochloride and urea denaturation. Proteins: Structure, Function, and Bioinformatics. 1, $81-89$ (1986).

5. Di Bilio, A.J., Crane, B.R., Wehbi, W.A., Kiser, C.N., Abu-Omar, M.M., Carlos, R.M., Richards, J.H., Winkler, J.R., Gray, H.B.: Properties of Photogenerated Tryptophan and Tyrosyl Radicals in Structurally Characterized Proteins Containing Rhenium(I) Tricarbonyl Diimines. J. Am. Chem. Soc. 123, 3181-3182 (2001).

6. Miller, J.E., Grădinaru, C., Crane, B.R., Di Bilio, A.J., Wehbi, W.A., Un, S., Winkler, J.R., Gray, H.B.: Spectroscopy and Reactivity of a Photogenerated Tryptophan Radical in a Structurally Defined Protein Environment. J. Am. Chem. Soc. 125, 14220-14221 (2003).

7. Gaertner, T.R., Kolodziej, S.J., Wang, D., Kobayashi, R., Koomen, J.M., Stoops, J.K., Waxham, M.N.: Comparative Analyses of the Three-dimensional Structures and Enzymatic Properties of $\alpha, \beta, \gamma$, and $\delta$ Isoforms of Ca2+-Calmodulin-dependent Protein Kinase II. J. Biol. Chem. 279, 12484-12494 (2004). 


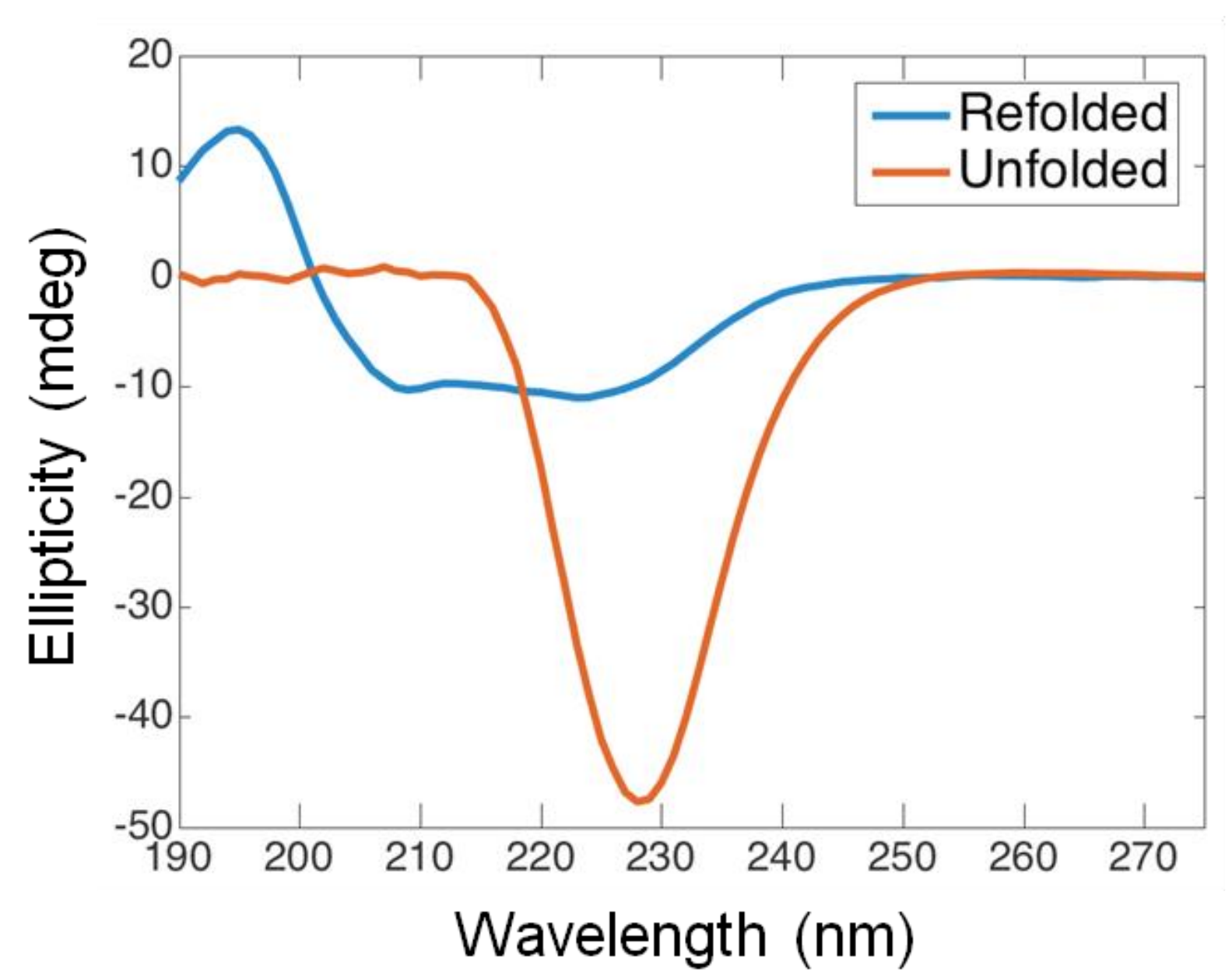

Figure S1: CD spectra of SNase in the unfolded state during purification (orange) and after refolding to the native structure via dialysis (blue). The curve with the significant negative peak at $230 \mathrm{~nm}$ is characteristic of an unfolded protein in urea-containing buffer. The curve with the positive peak at $195 \mathrm{~nm}$ and two negative peaks at $208 \mathrm{~nm}$ and $222 \mathrm{~nm}$ is characteristic of a folded protein containing alpha-helical and beta-sheet secondary structure. 


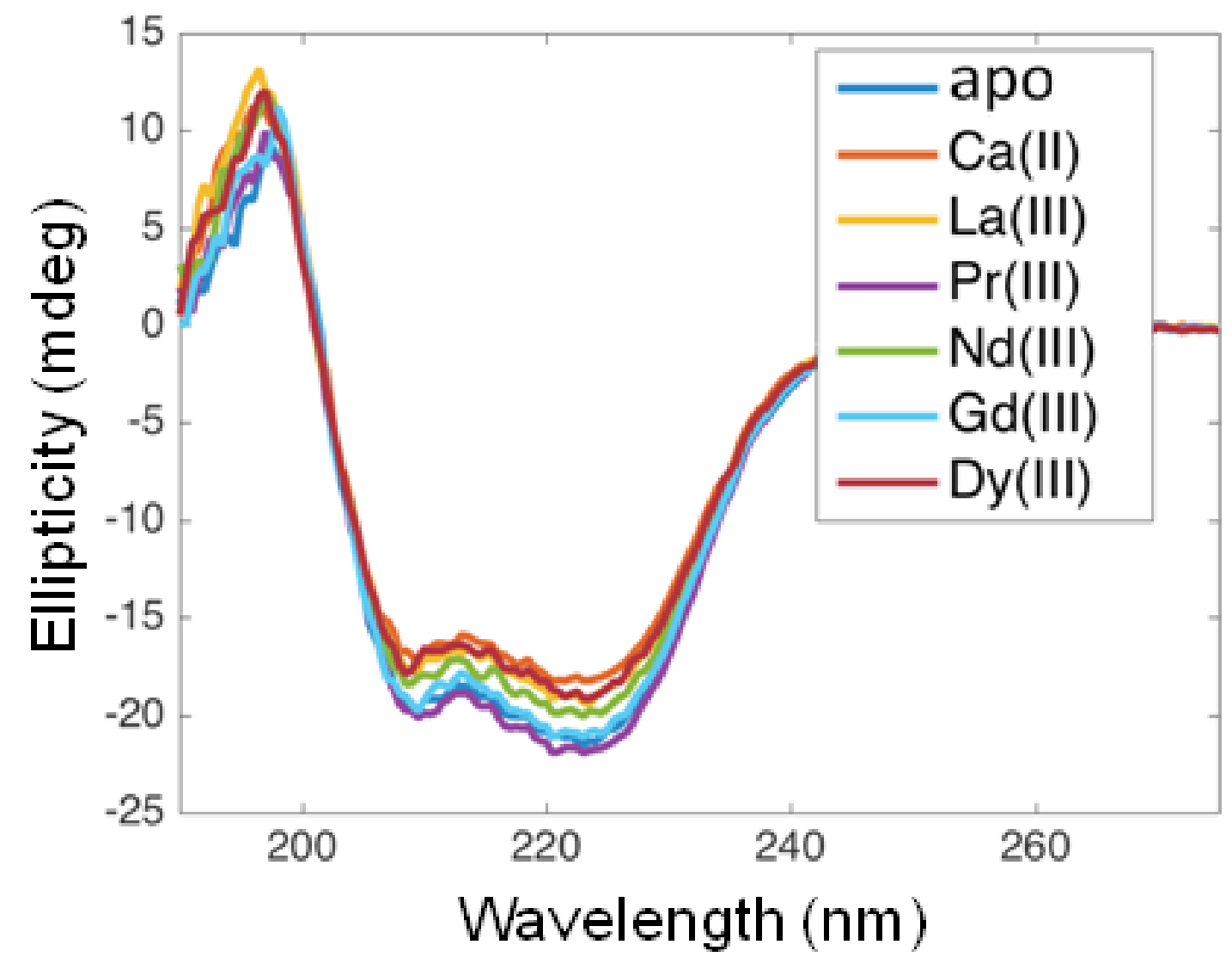

Figure S2: CD spectra (not normalized) of SNase bound to the native calcium, different lanthanide metals, and metal-free (apo). No differences were observed in the CD spectra of apo SNase compared to the metal complexes. 


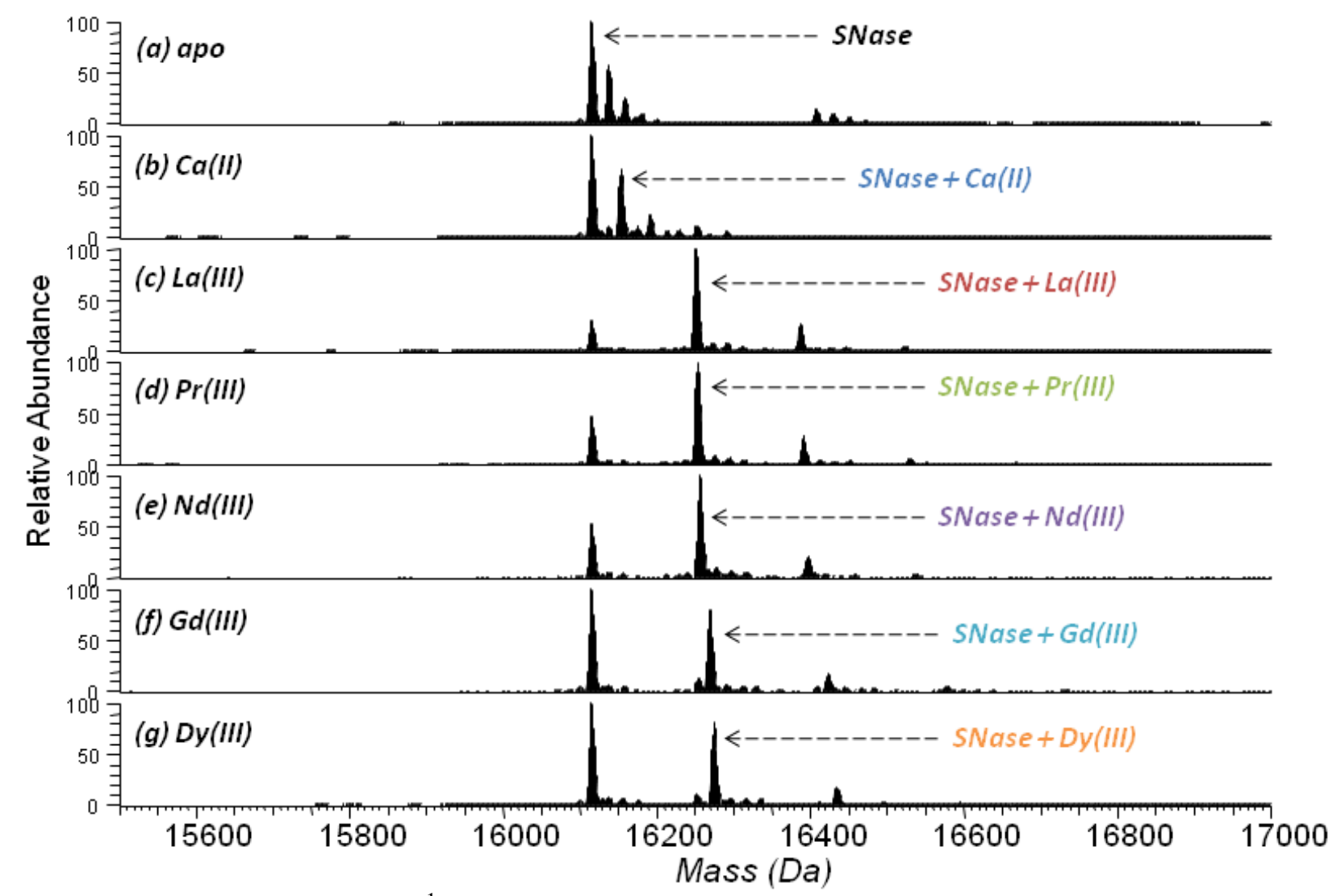

Figure S3: Deconvoluted $\mathrm{MS}^{1}$ spectra for SNase and the metal complexes. Deconvolution was achieved using the Xtract algorithm with a signal to noise ratio of 3. (a) apo SNase (16105.37 Da), (b) $\mathrm{Ca}$ (II) complex (16145.30 Da), (c) La(III) complex (16241.27 Da), (d) Pr(III) complex (16243.28 Da), (e) Nd(III) complex (16246.27 Da), (f) Gd(III) complex (16260.28 Da), and (g) Dy(III) complex (16265.28 Da). Monoisotopic masses are shown in parenthesis. 


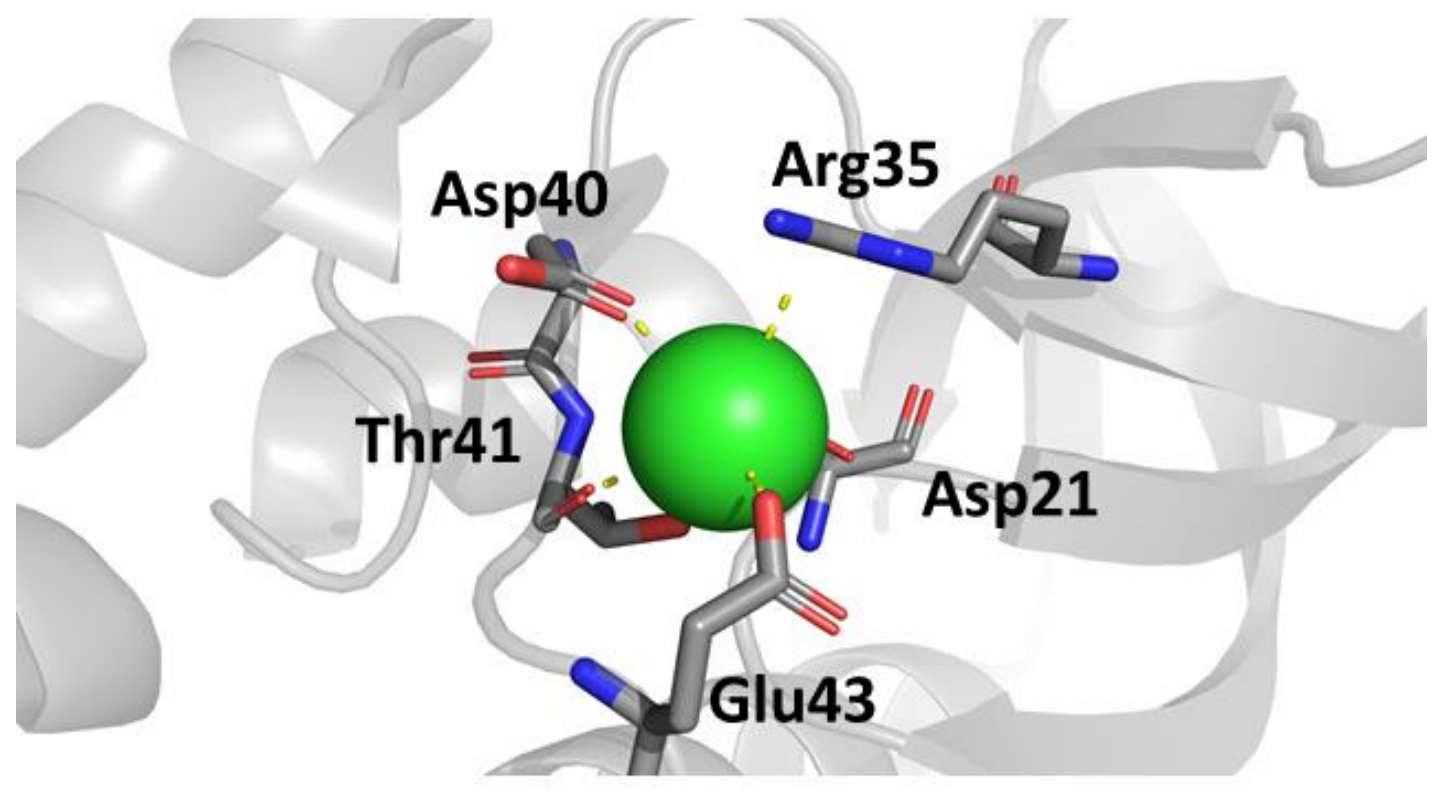

Figure S4: Expansion of the calcium binding region for SNase (PDB: 3BDC), showing the polar contacts (yellow) between the calcium ion (green) and the protein (sidechains). Polar contacts are shown to Asp 21, Arg 35, Asp 40, Thr41, and Glu43. 


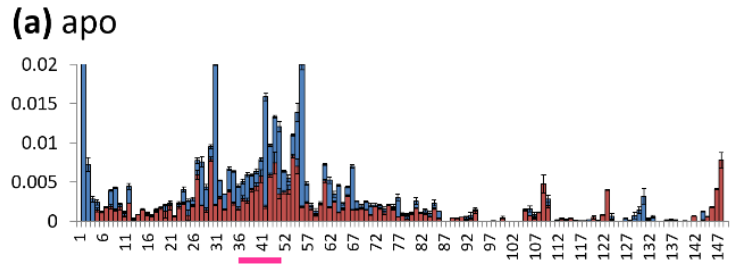

(b) $\mathrm{Ca}(\mathrm{II})$ apo

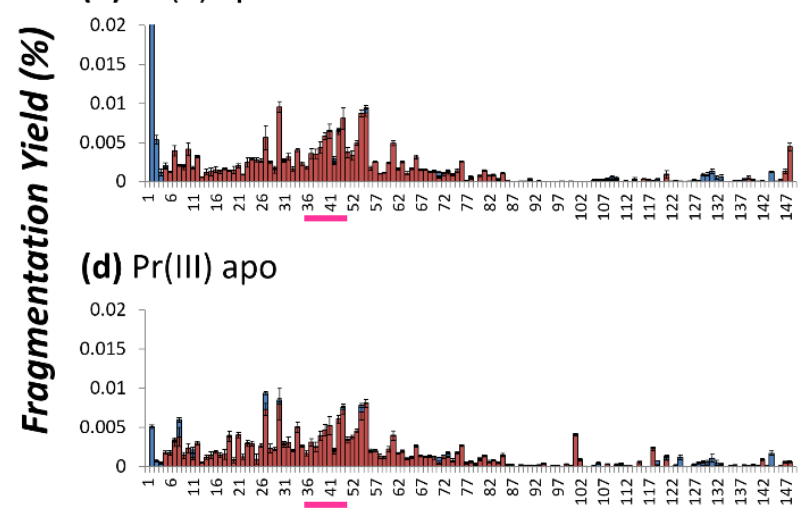

Residue Number (c) $\mathrm{Ca}$ (II) holo

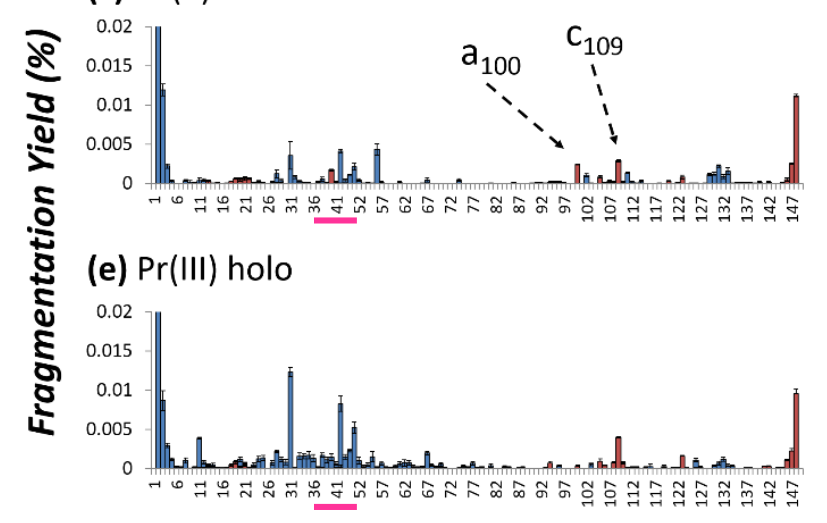

Residue Number

\section{N-terminal C C-terminal}

Figure S5: Distributions of the backbone cleavage positions that result in N- and C-terminal product ions of (a) apo SNase, (b) $\mathrm{Ca}$ (II)-coordinated SNase apo ions, (c) $\mathrm{Ca}$ (II)-coordinated SNase holo ions, (d) Pr(III)-coordinated SNase apo ions, and (e) Pr(III)-coordinated SNase holo ions produced via UVPD. Backbone cleavage positions that result in $\mathrm{N}$-terminal ions are shown in red and $\mathrm{C}$-terminal ions are shown in blue. The binding sites for $\mathrm{Ca}(\mathrm{II})$ and $\mathrm{Pr}(\mathrm{III})$ are demarcated by pink bars. 


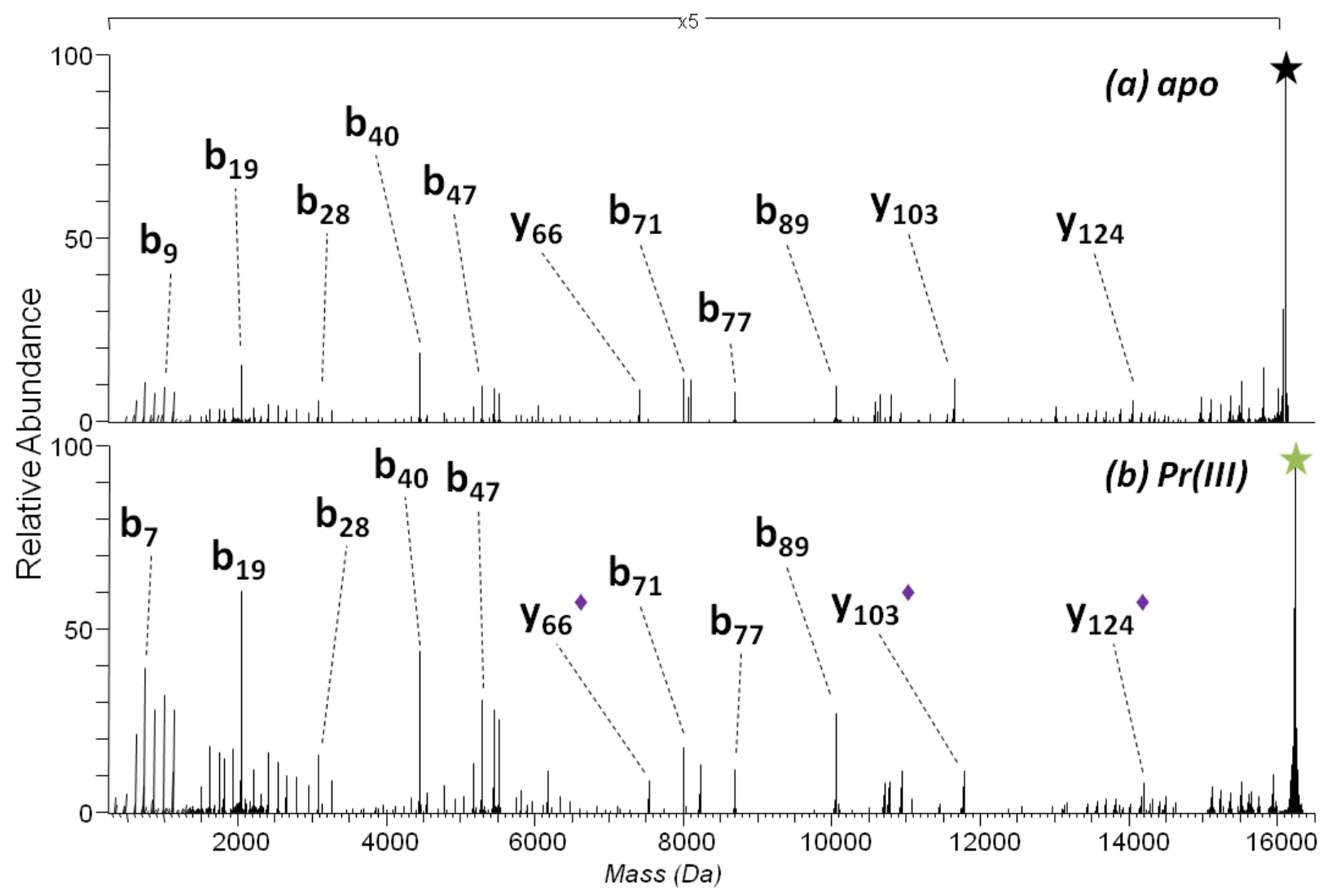

Figure S6: Deconvoluted $\mathrm{MS}^{2}$ mass spectra $(\mathrm{HCD}, \mathrm{NCE}=30,8+$ ) of (a) apo SNase and (b) the $\operatorname{Pr}(\mathrm{III})$ complex. A magnification factor of $5 \mathrm{X}$ was applied to the fragment ions. Holo fragment ions (i.e. retaining the metal ion) are denoted with a purple diamond in the label. 


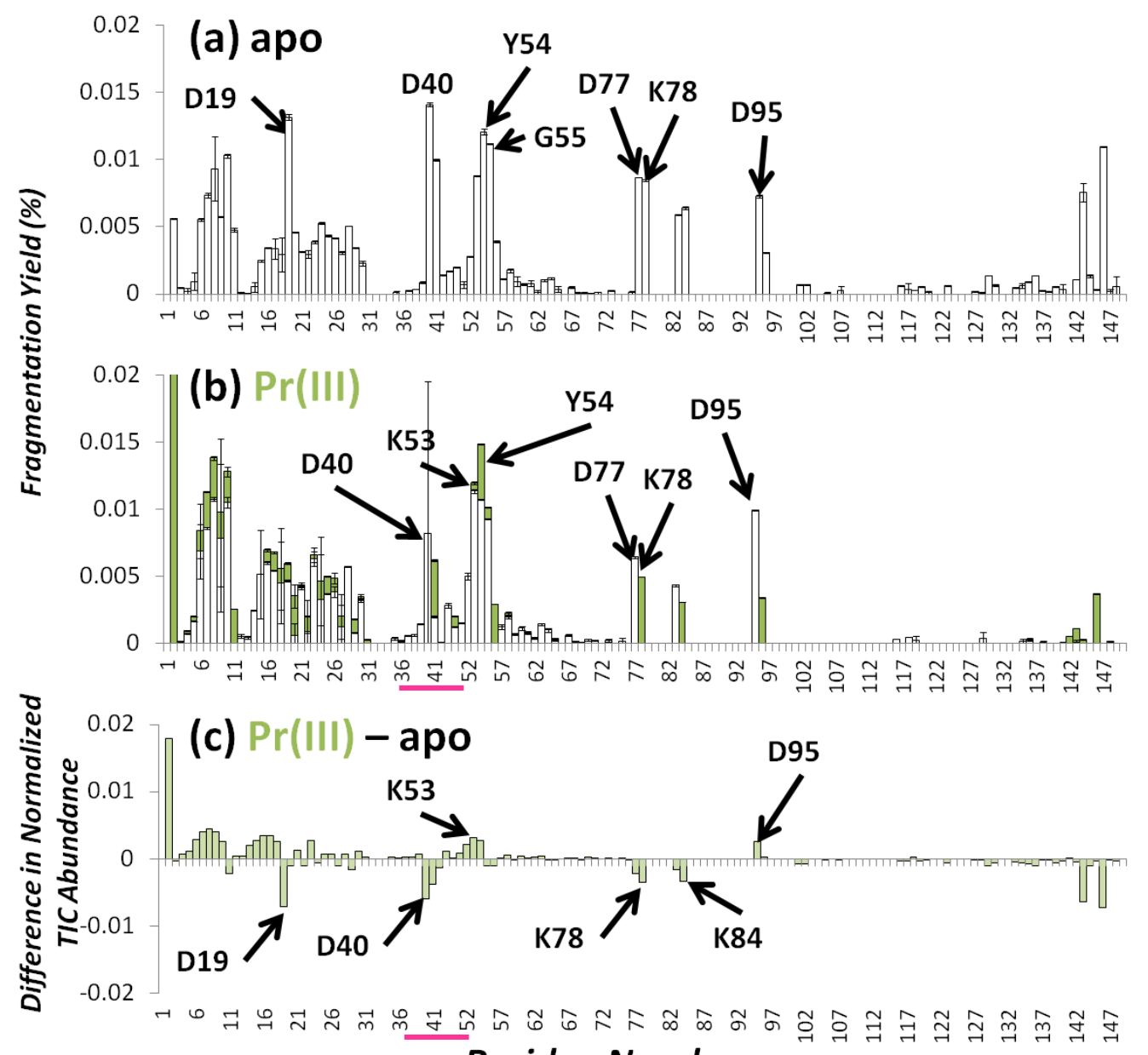

Residue Number

Figure S7: Backbone cleavage yields from HCD of (a) apo SNase (8+) and (b) $\operatorname{Pr}(\mathrm{III})$ complex $(8+)$, normalized to the TIC. The white bars represent apo fragment ions, and the green-shaded bars represent holo ions, and they are stacked to show the summed apo and holo fragment ions. (c) Difference plot showing the UVPD backbone cleavage yields of apo SNase (only apo fragment ions) subtracted from the backbone cleavage yields of the $\operatorname{Pr}(\mathrm{III})$ complex (summed holo and apo ions). Negative values represent regions of the protein backbone for which backbone cleavages of the metal complexes are suppressed relative to the apo-protein. Selected residues are labeled for clarity. The binding sites for $\operatorname{Pr}(\mathrm{III})$ are demarcated by pink bars 


\section{(a) apo SNase}

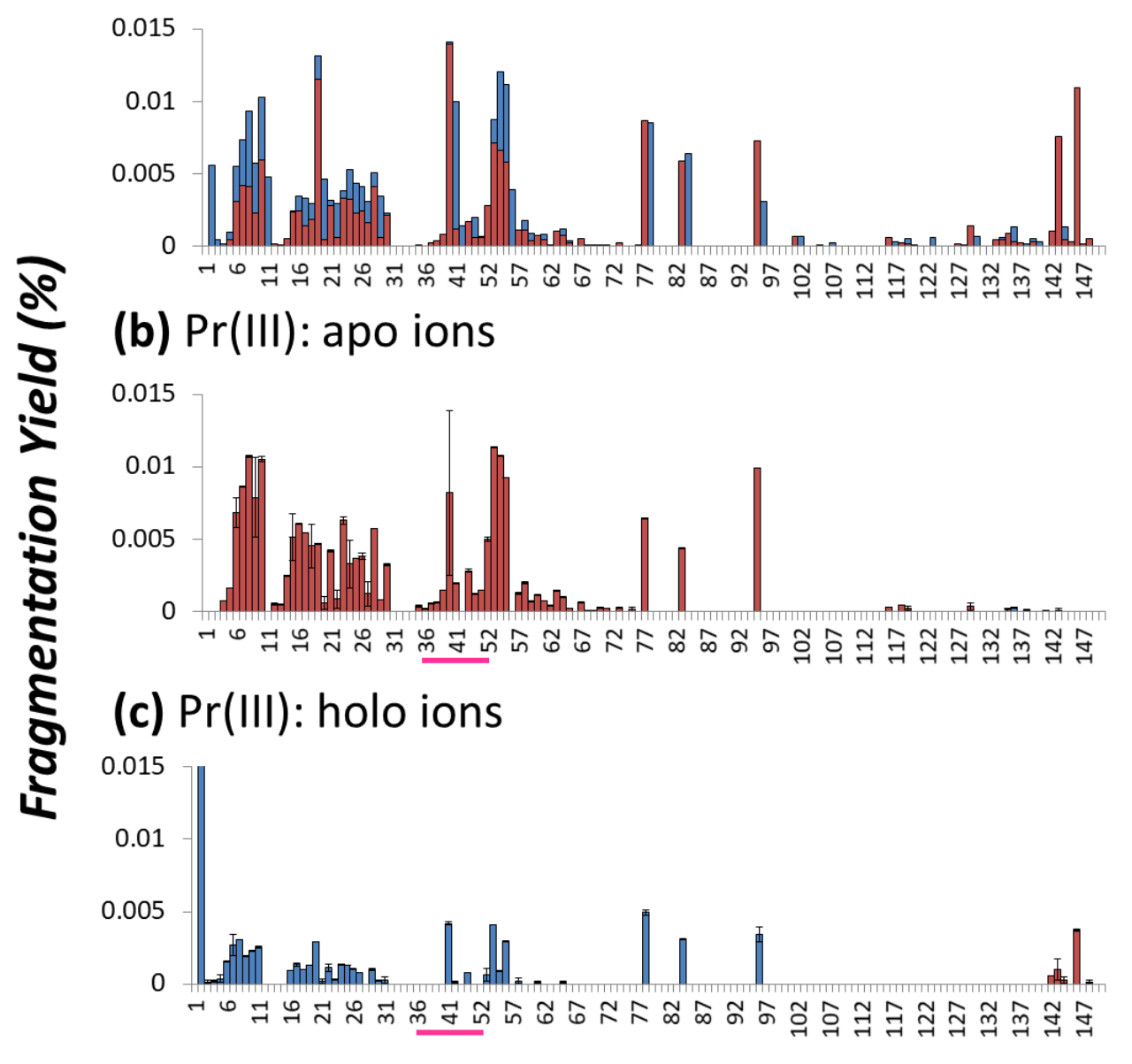

\section{Residue Number}

\section{N-terminal $\quad$ C-terminal}

Figure S8: Distribution of backbone cleavage positions that result in $\mathrm{N}$ - and C-terminal product ions of (a) apo SNase (8+), (b) apo fragment ions of Pr(III)-bound SNase (8+), and (c) holo fragment ions of $\operatorname{Pr}(\mathrm{III})$-bound SNase $(8+)$ produced via HCD. Backbone cleavage sites that result in $\mathrm{N}$-terminal ions (ions containing the $\mathrm{N}$-terminus of the protein) are shown in red, and $\mathrm{C}$-terminal ions (ions containing the $\mathrm{C}$-terminus of the protein) are shown in blue. The binding sites for $\operatorname{Pr}(\mathrm{III})$ are demarcated by pink bars. 


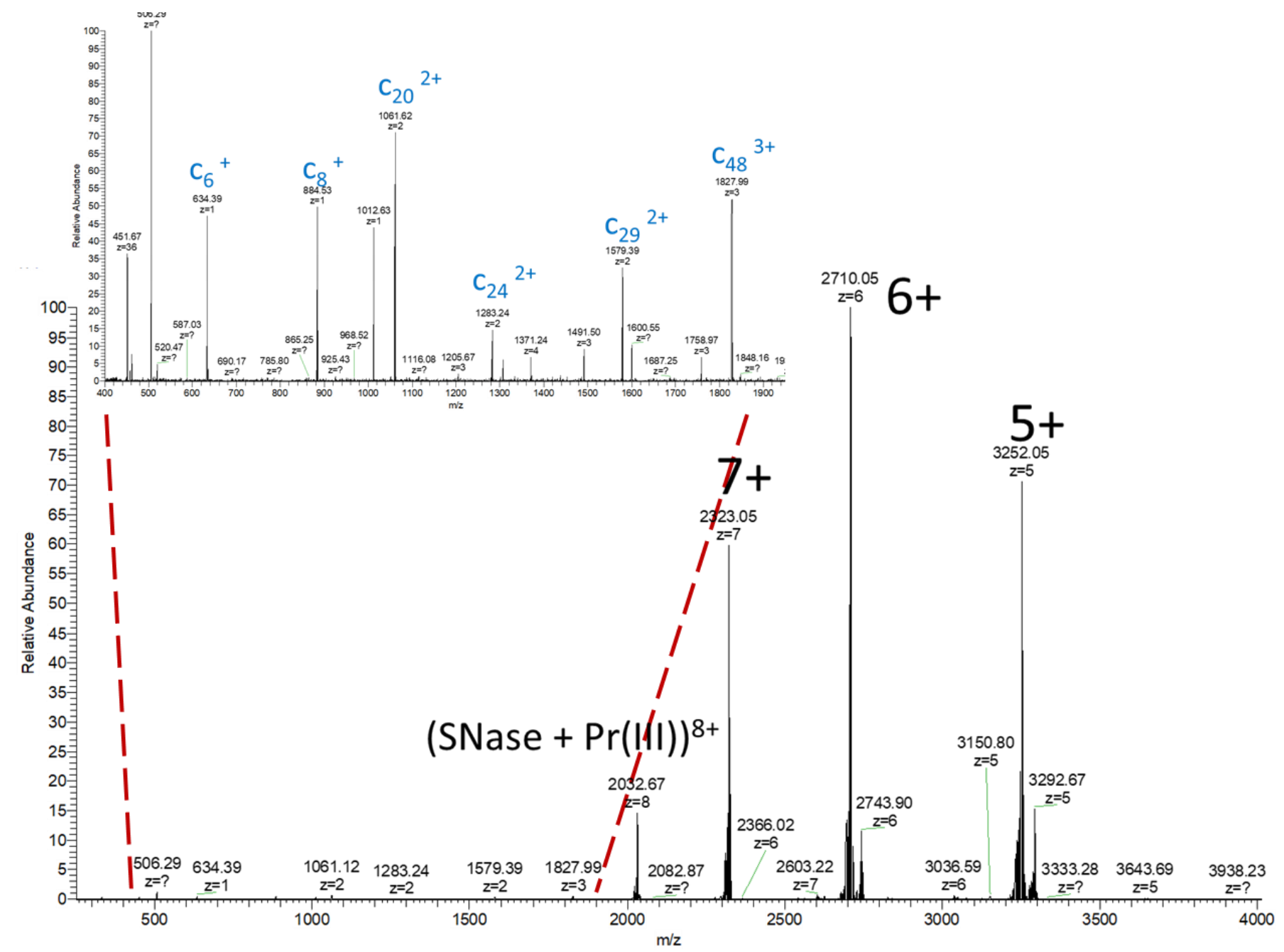

Figure S9: ETD MS ${ }^{2}$ mass spectrum (10 ms) of the SNase:Pr(III) complex (8+). The inset shows a magnified region of the spectrum displaying apo sequence ions of very low abundance. 


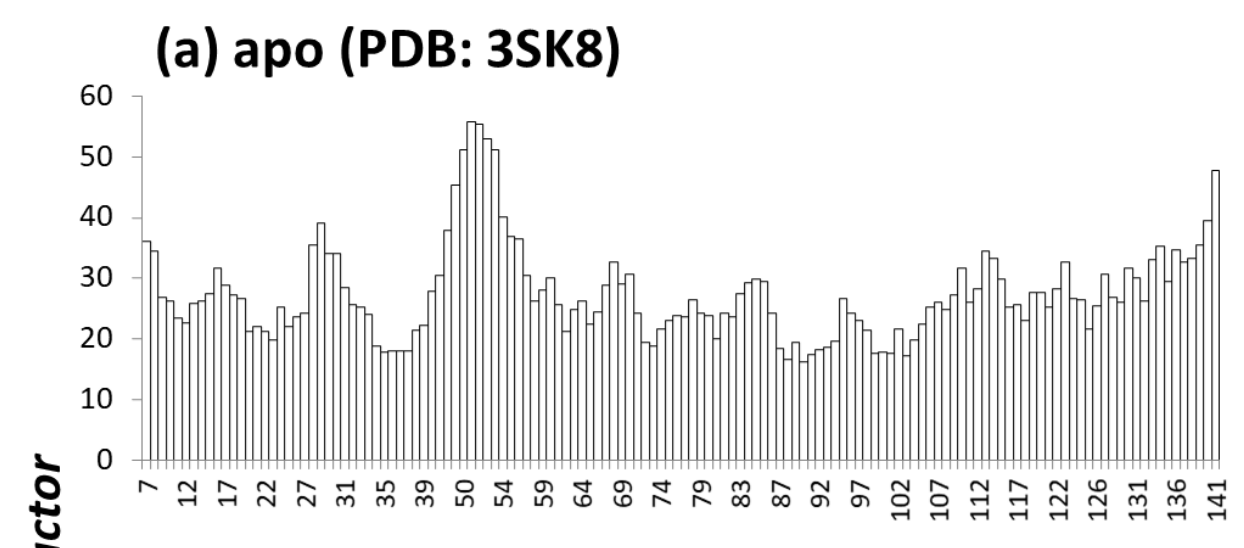

\section{(b) $\mathrm{Ca}$ (II) (PDB: 3BDC)}

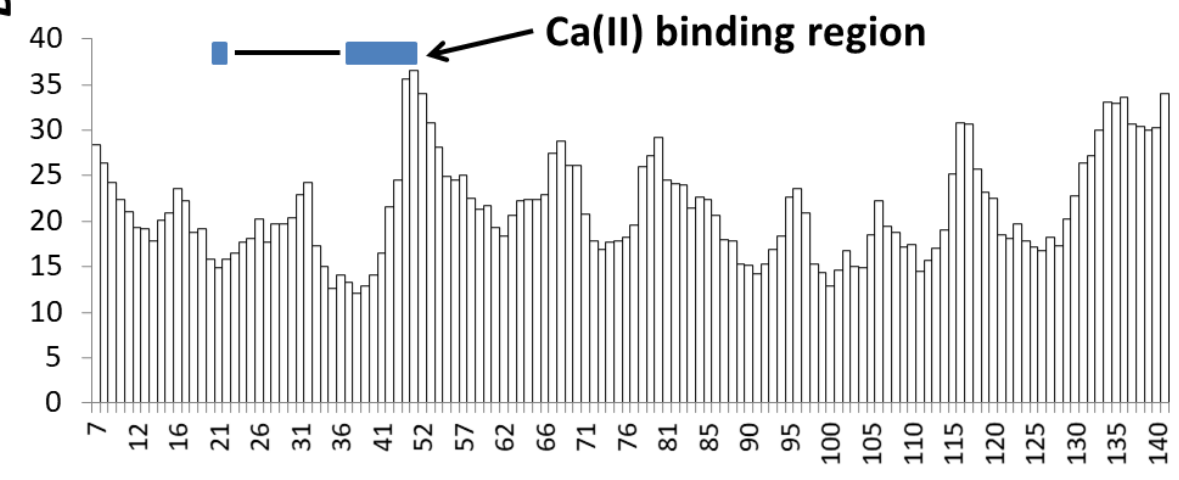

(c) Difference plot, apo- $\mathrm{Ca}$ (II) SNase

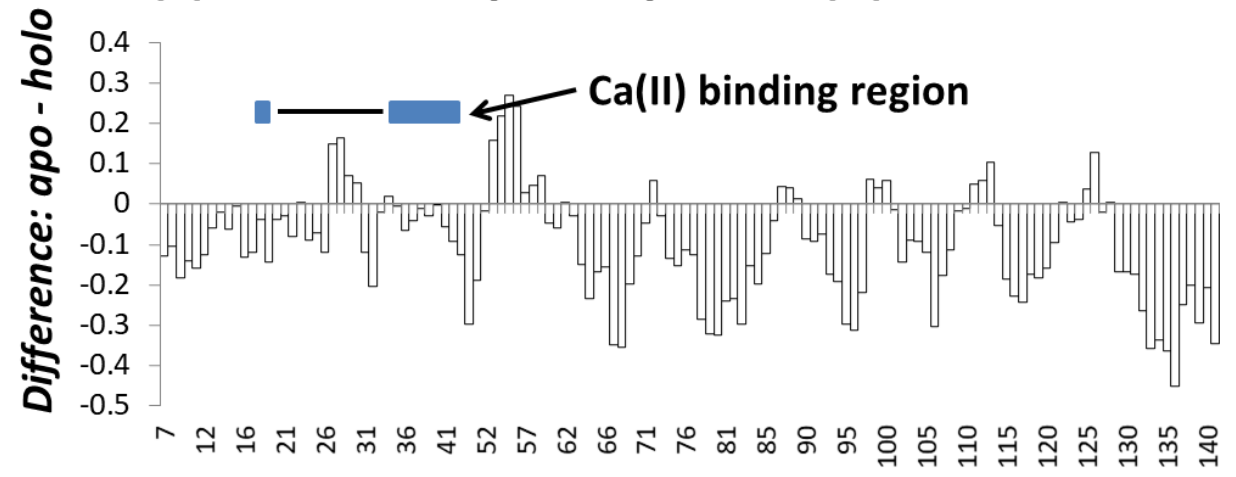

\section{Residue Number}

Figure S10: B-factors of (a) apo SNase (PDB: 3SK8) and (b) Ca(II)-bound SNase (PDB: 3BDC) plotted against the protein sequence. Regions of high structural flexibility have higher B-factors, and more rigid or less flexible regions have low values. (c) Difference plot between normalized B-factors (apo SNase - Ca(II) SNase). Positive values in the difference plot suggest greater structural flexibility for apo SNase and negative values suggest greater structural flexibility for $\mathrm{Ca}$ (II) SNase. The Ca(II) binding site is highlighted in blue. The crystal structures only reported the protein sequence starting at residue Leu7. 


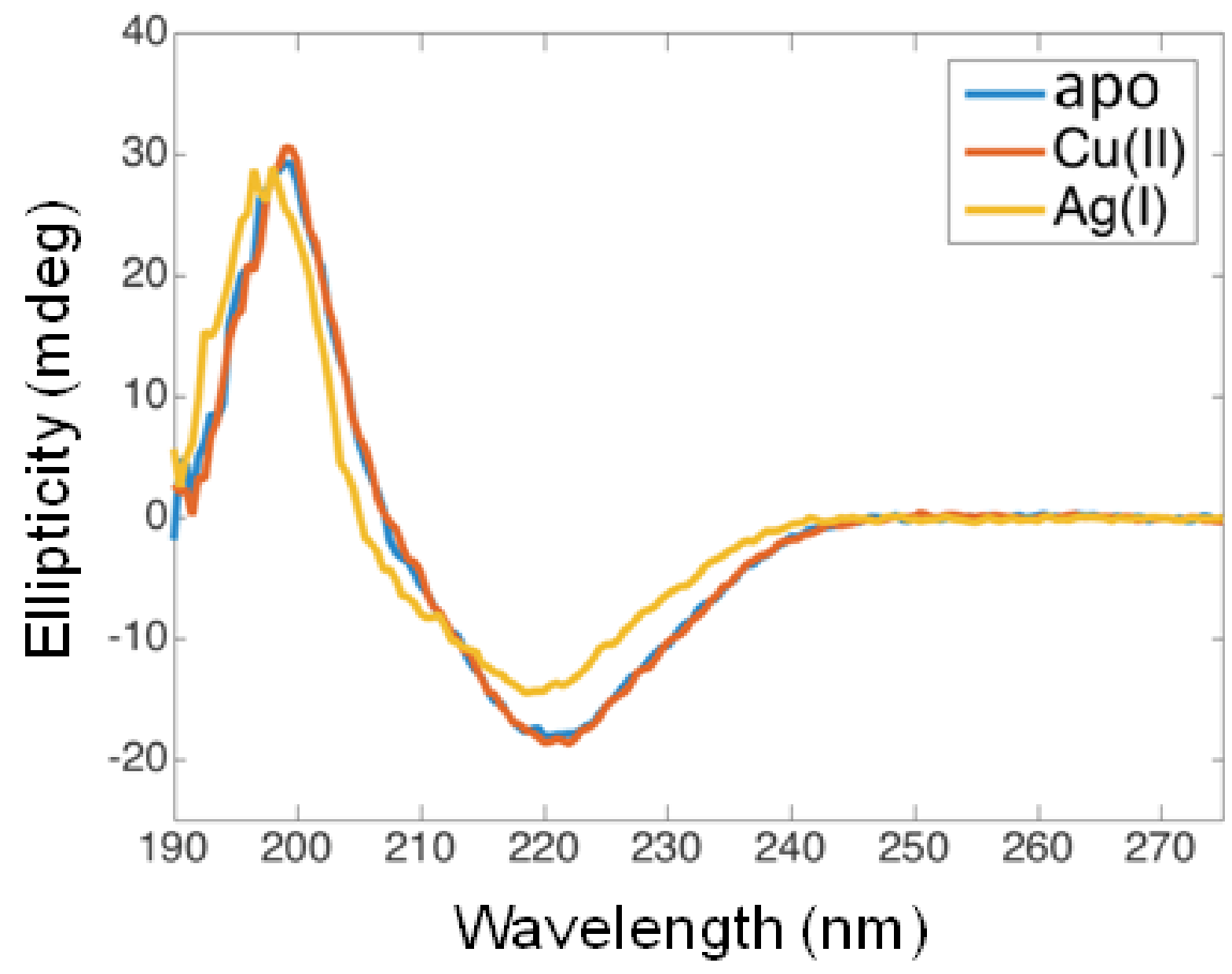

Figure S11: CD spectra (not normalized) of apo azurin or azurin bound to copper (II) or silver (I). No differences were observed in the CD spectra of apo-azurin (blue) and the $\mathrm{Cu}$ (II) complex (orange). The $\operatorname{Ag}(\mathrm{I})$ complex exhibits a blue-shift in the positive peak as well as the appearance of a slight negative peak at $\sim 208 \mathrm{~nm}$, both of which are characteristics of increased alpha-helical character. 


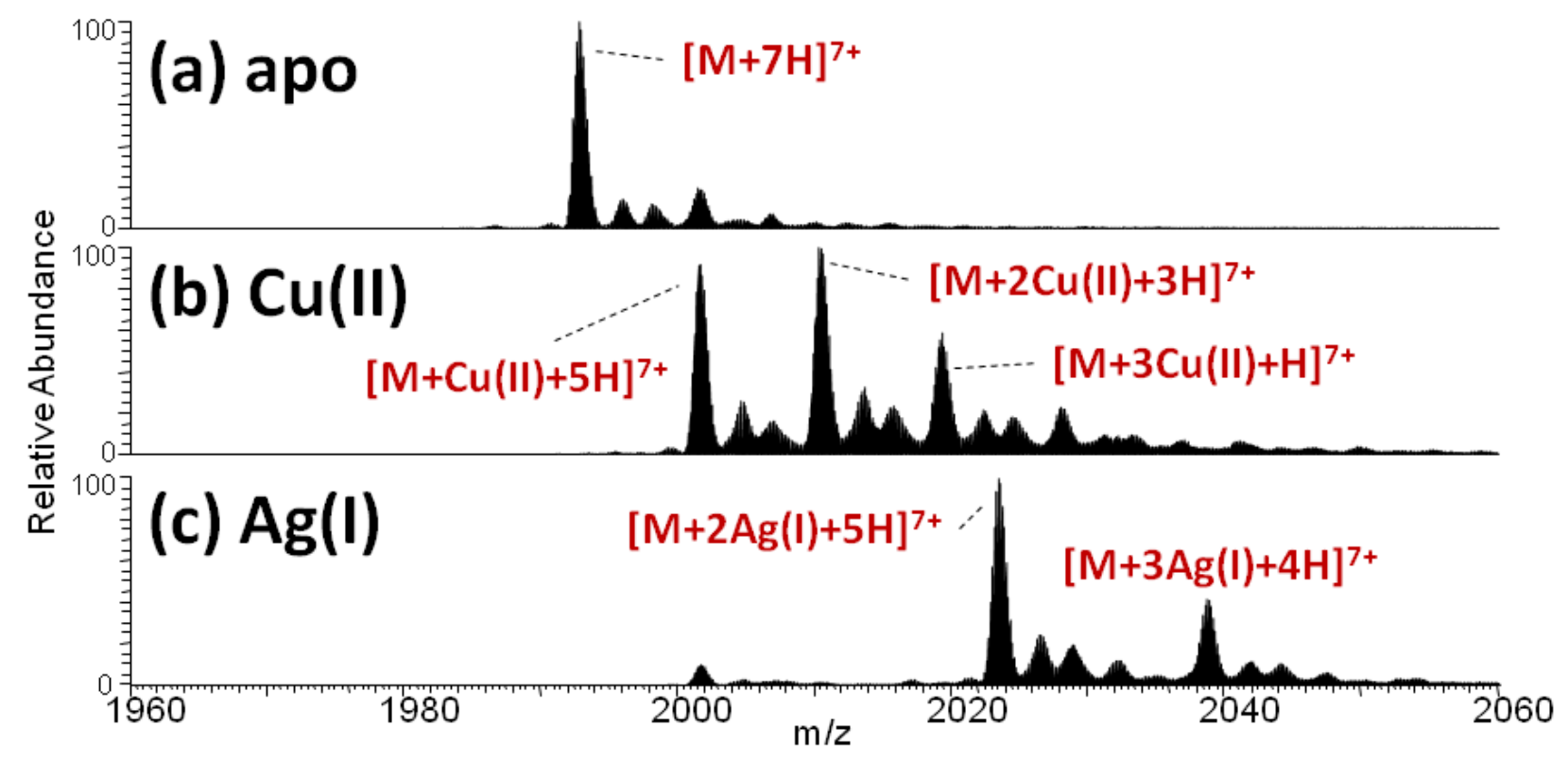

Figure S12: ESI mass spectra of solutions containing azurin: (a) no metal, and metal salts (b) $\mathrm{Cu}(\mathrm{II})$ and (c) $\mathrm{Ag}(\mathrm{I})$. All solutions contain $120 \mathrm{mM}$ ammonium acetate. 
(a) apo

Sequence coverage: $94 \%$

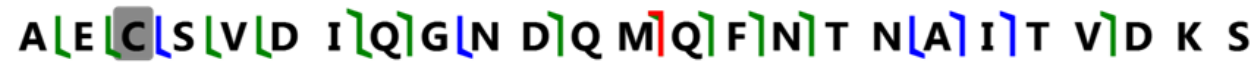

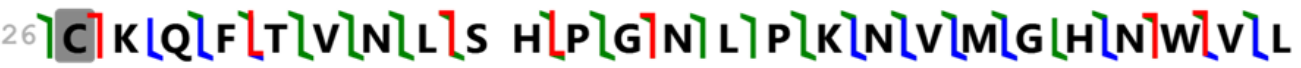

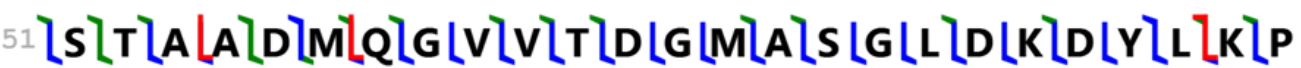

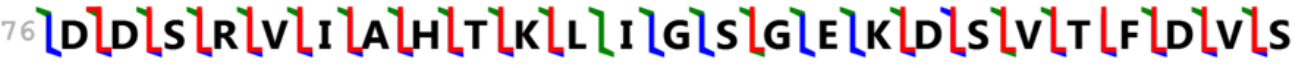

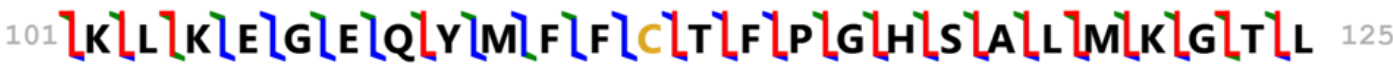
${ }_{126}\lfloor T \backslash L \backslash K$

(b) $\mathrm{Cu}$ (II) Sequence coverage: $83 \%$

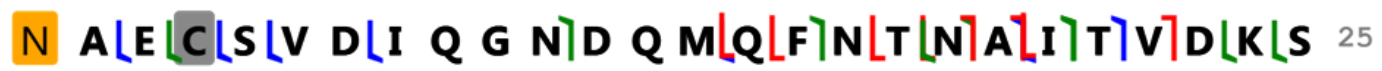

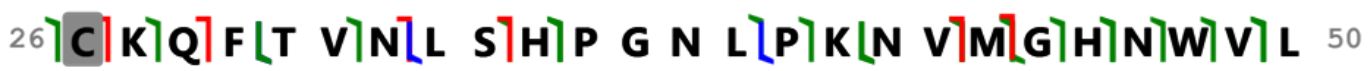

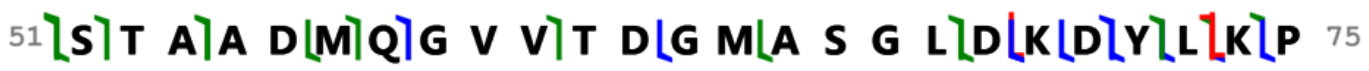

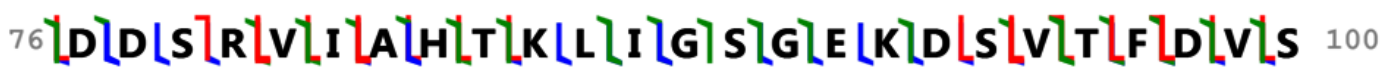

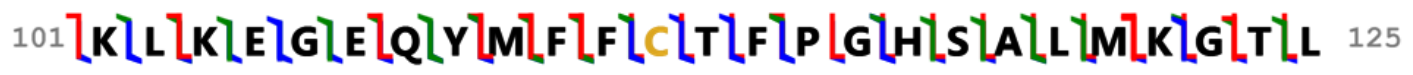
$\left.{ }_{126}\lfloor\mathbf{L}\rceil \mathbf{L}\right\rceil K$

(c) $\mathrm{Ag}(\mathrm{I}) \quad$ Sequence coverage: $81 \%$

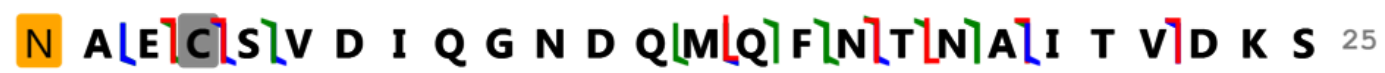

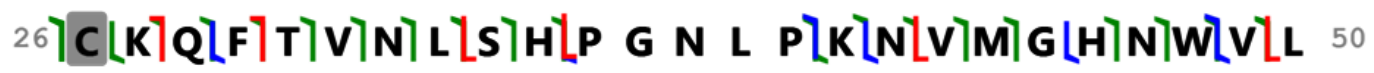

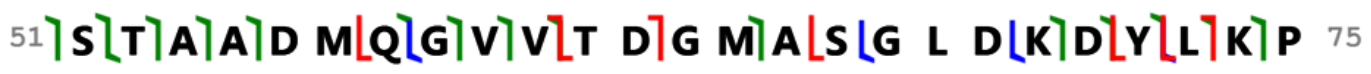

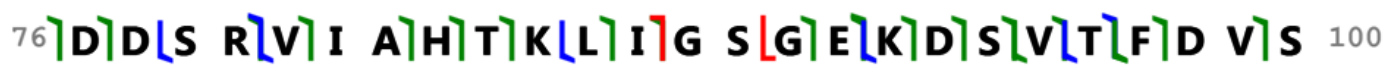

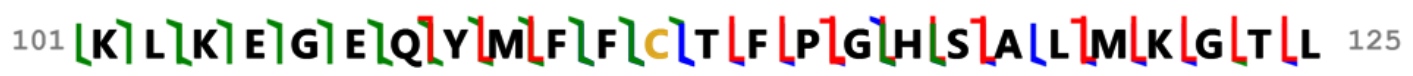
${ }_{126}$ LT LLLK

Figure S13: UVPD sequence maps for the 7+ charge states of (a) apo-azurin with $94 \%$ sequence coverage, (b) $\mathrm{Cu}$ (II) complexes with $83 \%$ sequence coverage, and (c) $2 \mathrm{Ag}$ (I) complexes with $81 \%$ sequence coverage. $a / x$ (green), $b / y$ (blue), and $c / z^{\bullet}$ (red) fragment ions are indicated by the colorcoded flags. The gray-shaded cysteines indicate the presence of a disulfide bond. 

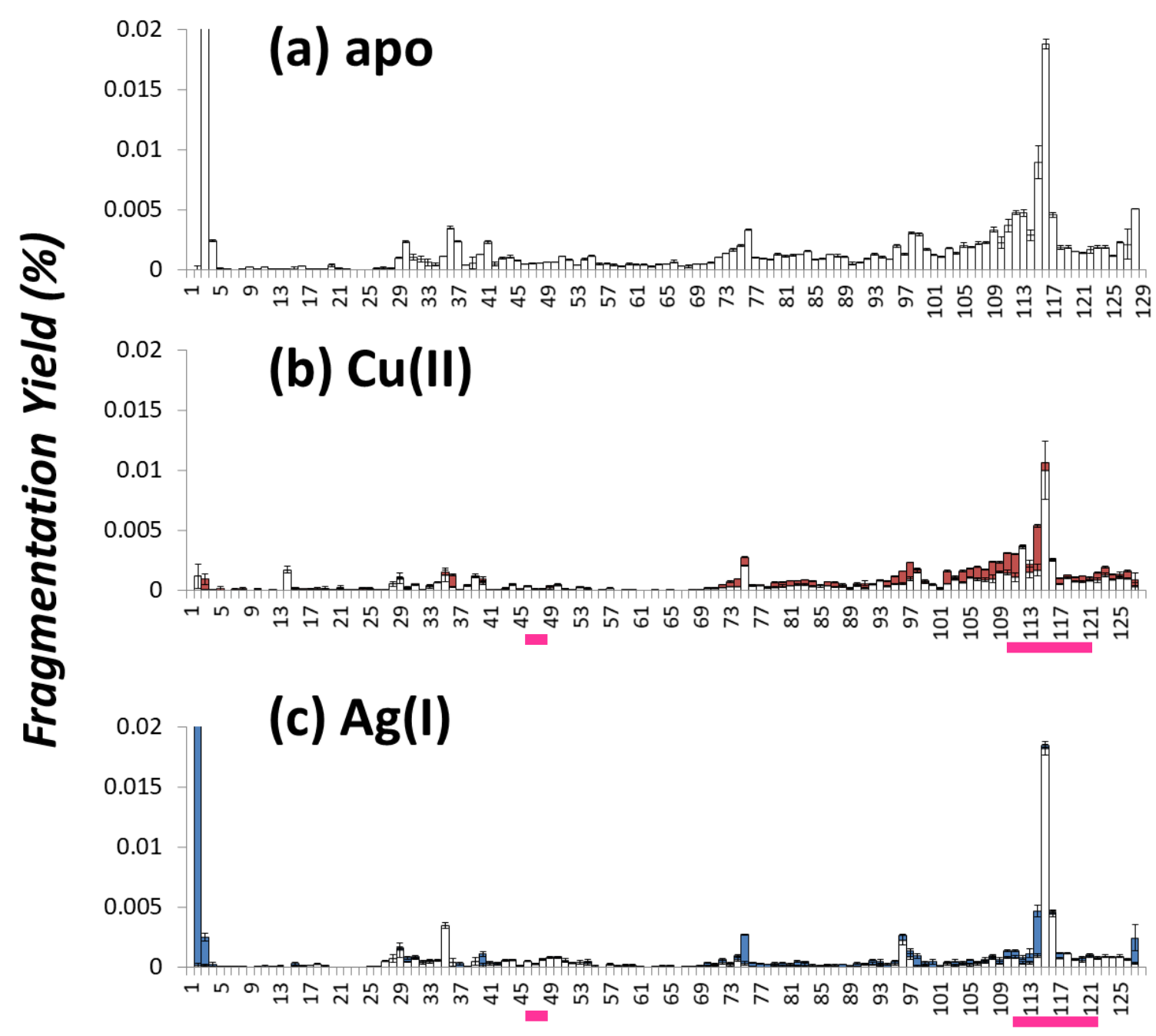

\section{Residue Number}

Figure S14: Backbone cleavage yields from UVPD of (a) apo azurin (7+) versus (b) $\mathrm{Cu}(\mathrm{II})$ complex (7+) and (c) $2 \mathrm{Ag}(\mathrm{I})$ complex (7+), normalized to the TIC. The white bars represent apo fragment ions, and the colored bars represent holo ions, and they are stacked to show the summed apo and holo fragment ions. The binding sites for copper and silver are demarcated in pink bars. 
(a)

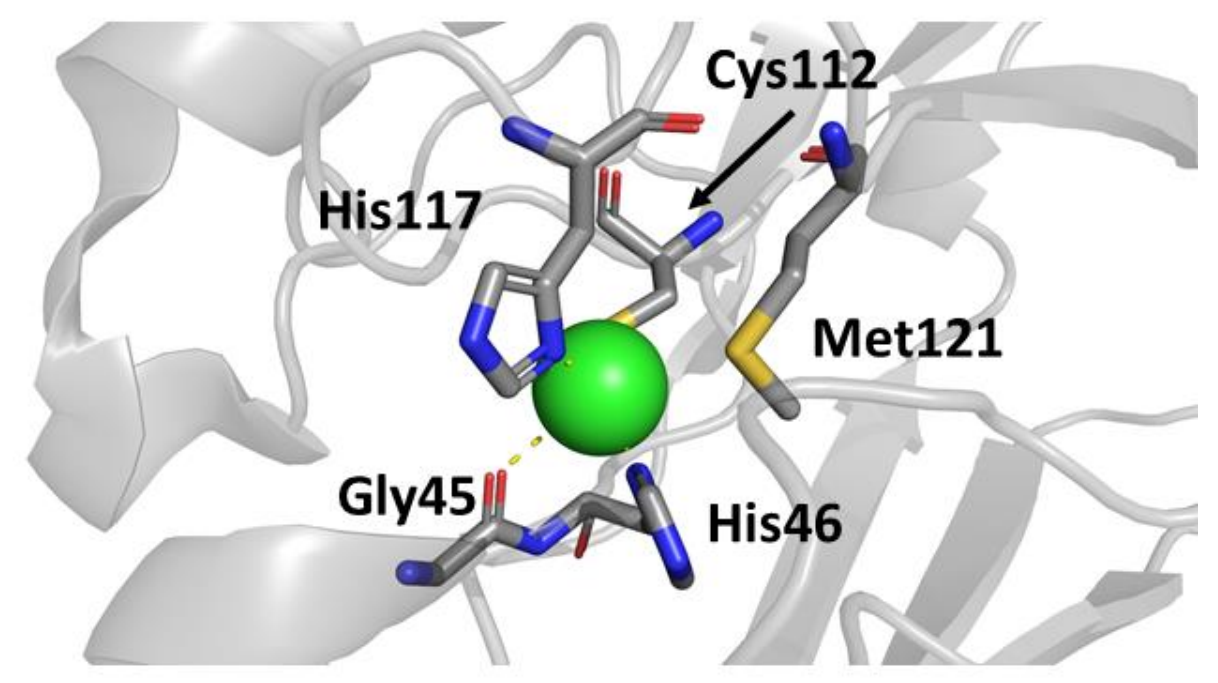

(b)

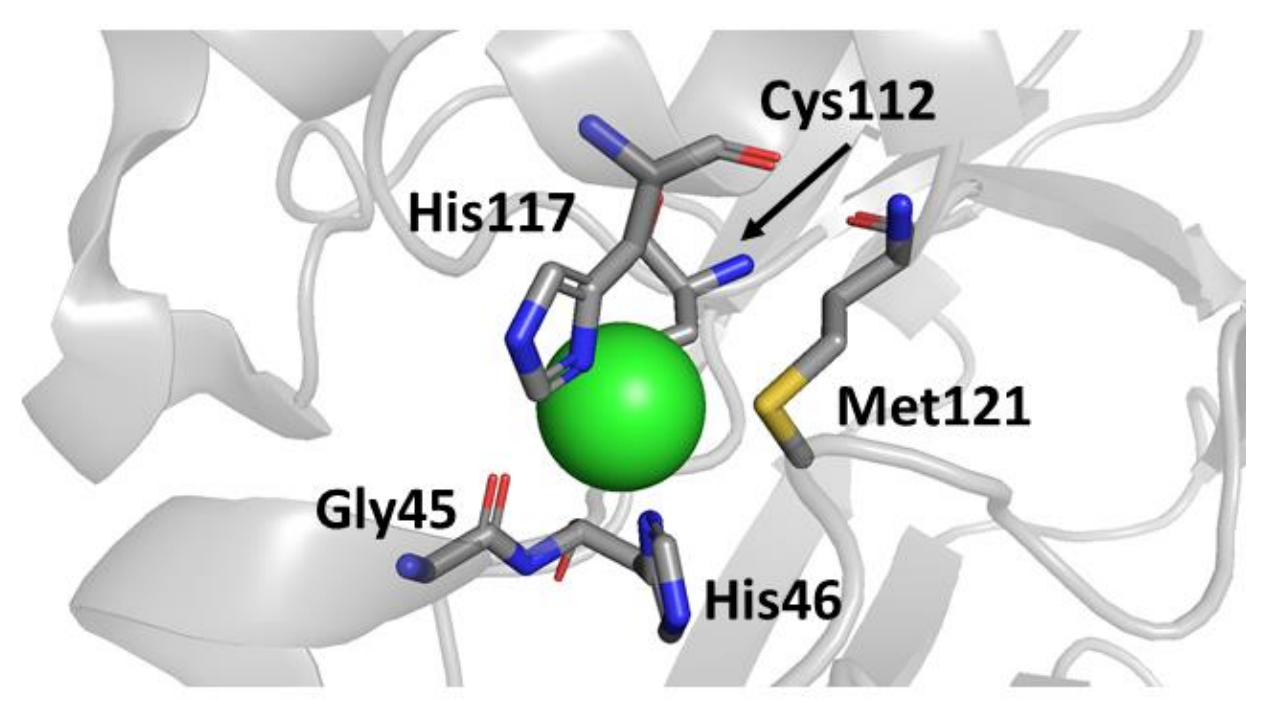

Figure S15: (a) Expansion of the copper binding region for azurin (PDB: 4AZU), showing the polar contacts (yellow) between the copper ion (green) and the protein (side-chains). Polar contacts are shown between Gly45 (carbonyl), His46, and His117. Cys112 and Met121 are also in close spatial proximity to the $\mathrm{Cu}(\mathrm{II})$ ion. (b) Expansion of the silver binding region for azurin (PDB: 3UGE), showing the lack of polar contacts (absence of yellow contact lines) between the silver ion (green) and the protein backbone. No polar contacts are identified, but the same five residues comprise a similar binding pocket to the $\mathrm{Cu}(\mathrm{II})$ :azurin complex. 

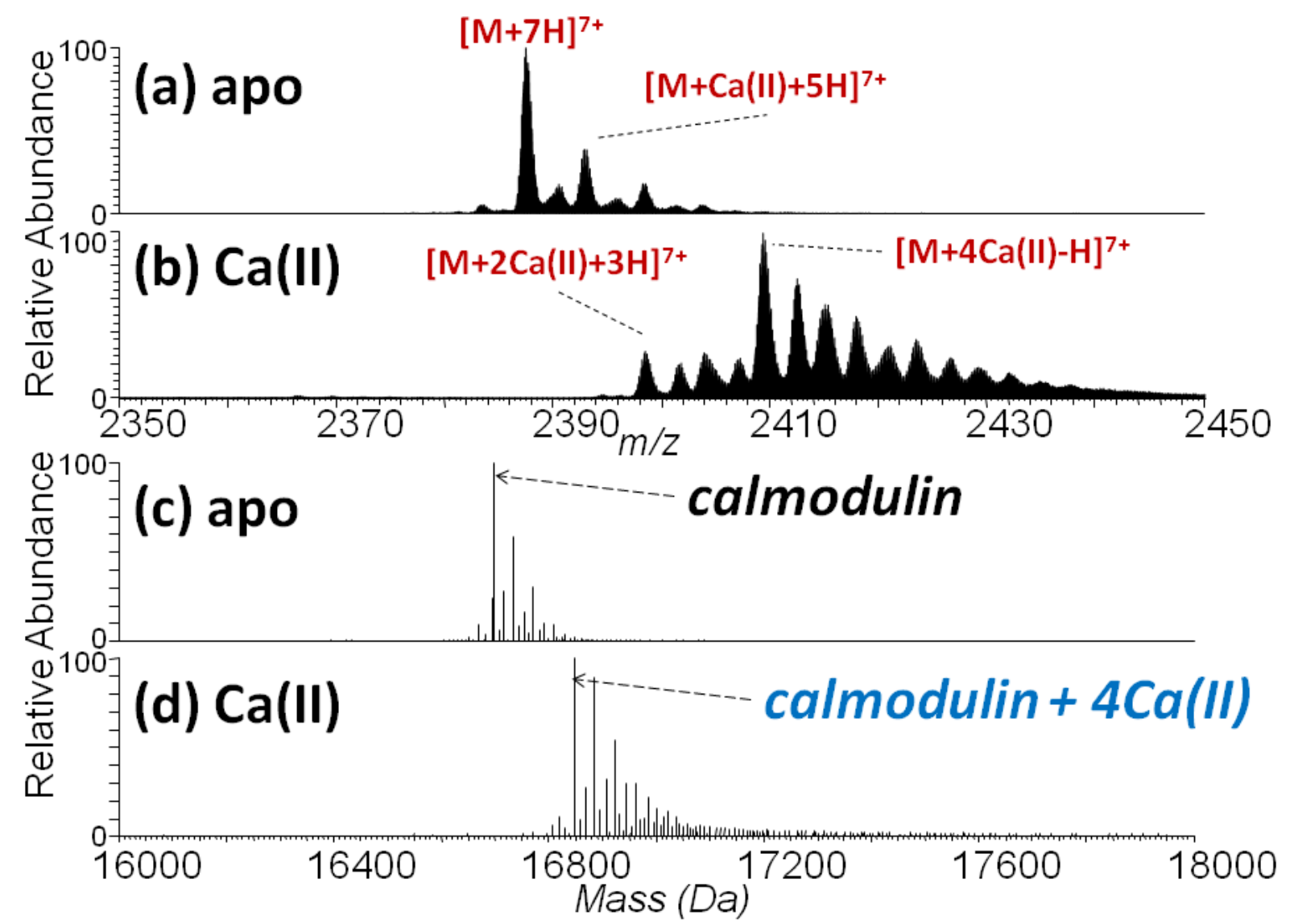

Figure S16: ESI mass spectra of solutions containing calmodulin: (a) no metal, and (b) calcium acetate. All solutions contain $120 \mathrm{mM}$ ammonium acetate. Deconvoluted $\mathrm{MS}^{1}$ spectra for calmodulin and the calcium complex: (c) apo calmodulin (16696.94 Da) and (d) Ca(II) complexes (4 Ca, 16847.53 Da). Monoisotopic masses are shown in parenthesis. 


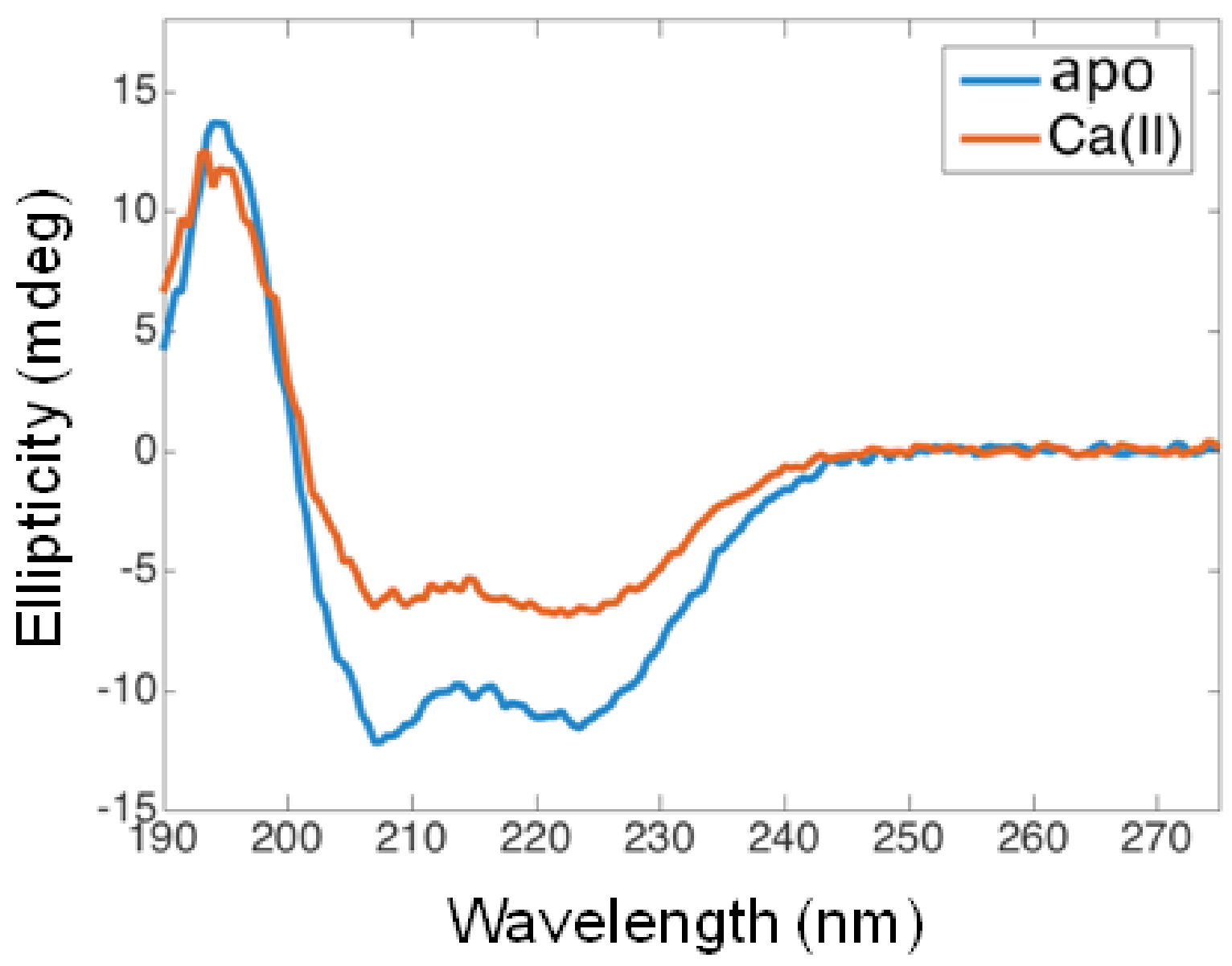

Figure S17: CD spectra (not normalized) of apo calmodulin (blue) and calmodulin bound to calcium (orange). Similar spectral features were observed in the CD spectra of apo-calmodulin and the $\mathrm{Ca}$ (II) complexes, indicating that both contain mostly alpha-helical secondary structure. The CD measurements do not reveal differences in tertiary structures between apo and holo calmodulin. 


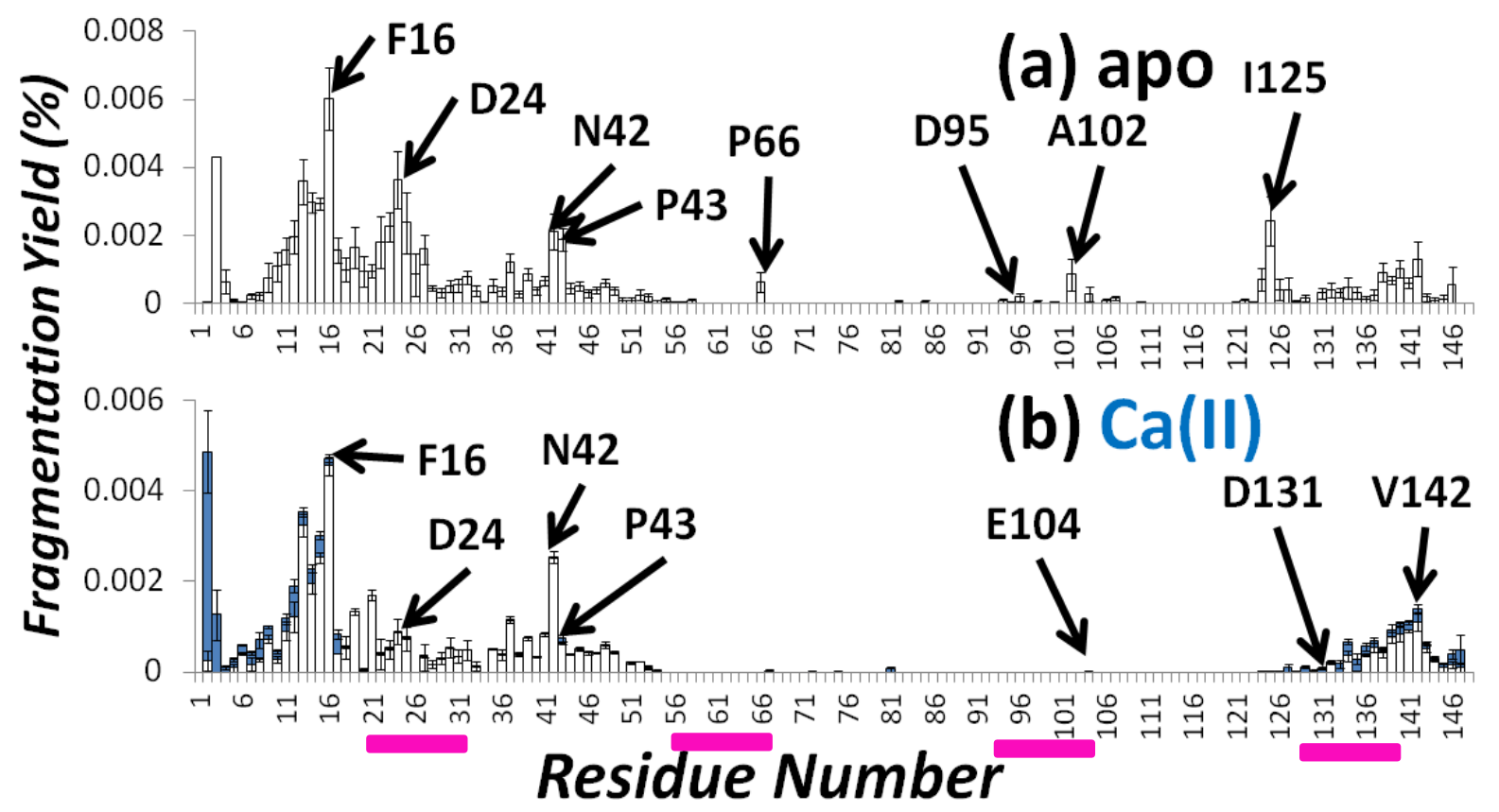

Figure S18: Backbone cleavage yields from UVPD of (a) apo calmodulin and (b) $\mathrm{Ca}$ (II) complexes (7+), normalized to the TIC. The white bars represent apo fragment ions, and the blueshaded bars represent holo ions, and they are stacked to show the summed abundances. The binding sites for calcium are demarcated in pink bars. 


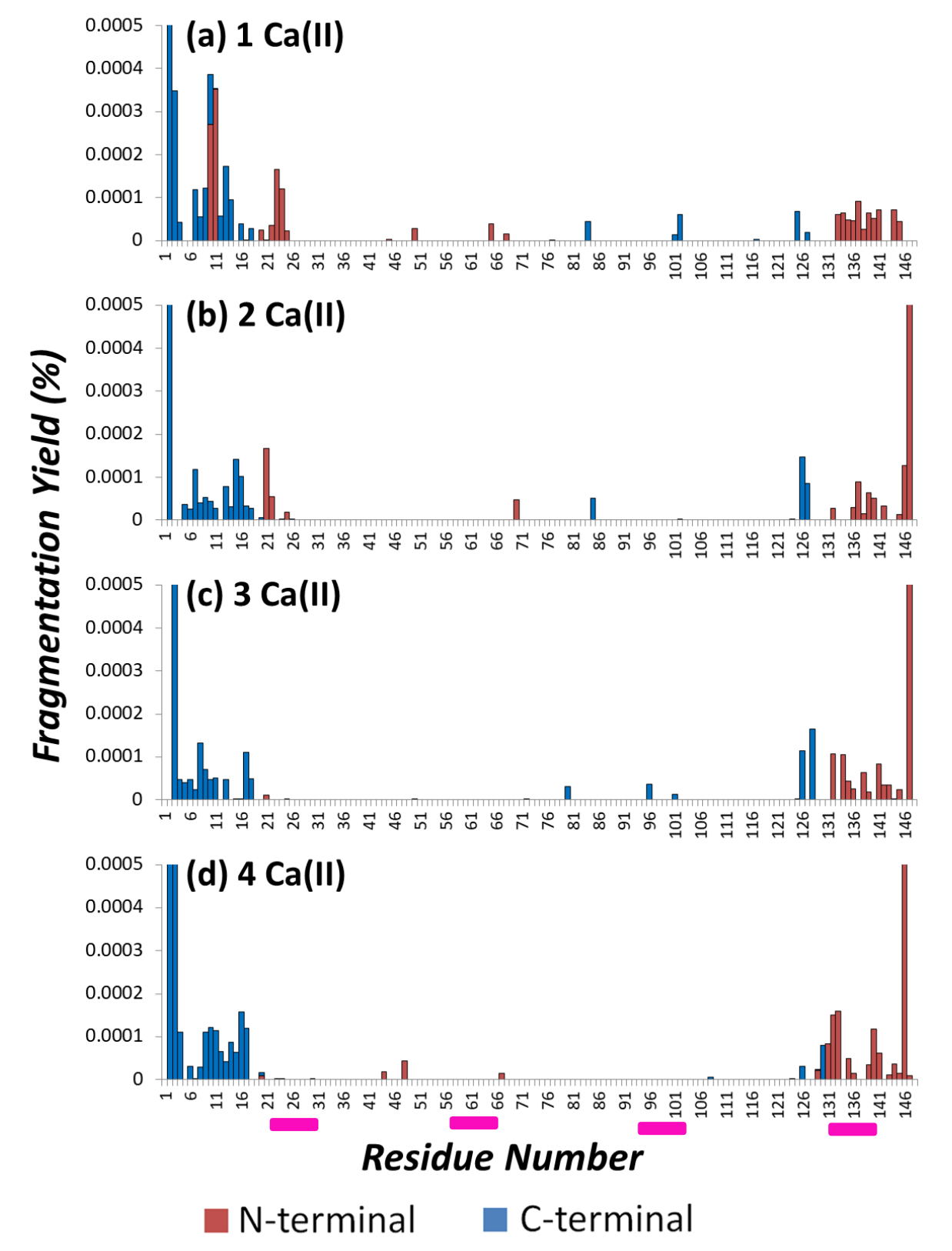

Figure S19: Distribution of backbone cleavage positions that result in holo fragment ions from UVPD of $\mathrm{Ca}(\mathrm{II})$ complexes (7+), showing the number of metal ions retained: (a) $1 \mathrm{Ca}$ (II), (b) 2 $\mathrm{Ca}(\mathrm{II})$, (c) $3 \mathrm{Ca}$ (II), and (d) $4 \mathrm{Ca}$ (II). Backbone cleavage sites that result in N-terminal ions are shaded red, and C-terminal ions are shaded blue, and they are stacked to show the summed abundances. The binding sites for calcium are demarcated in pink bars. 


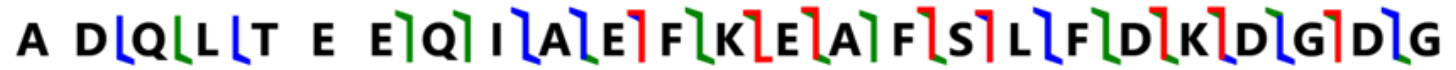

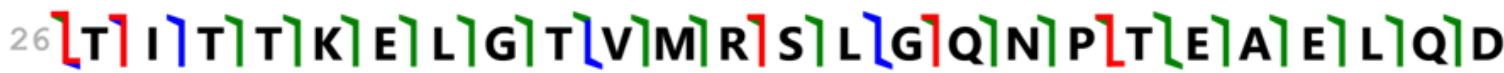

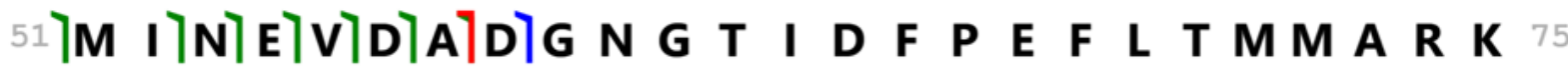
${ }^{76}$ M]K D T D S E E E I R E A F RLV F DLKLDLG NLG YLI

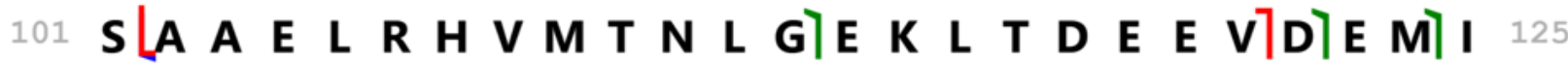

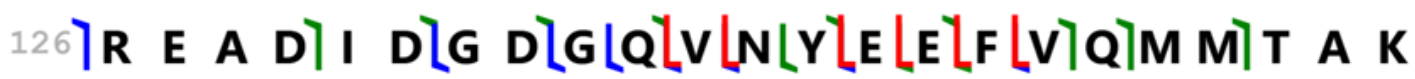

(b) $\mathrm{Ca}$ (II) Sequence coverage: $43 \%$

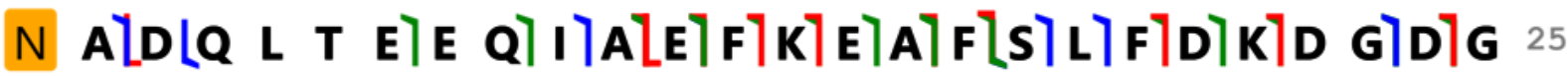

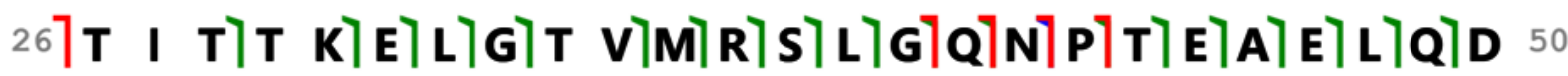
51 M) I N N 76 M K D T D S E ELE I R E A F R V F D K D G N G Y I 100 101 LS A ALE L R H V M T N L G E K L T D E E V D E MLI 125

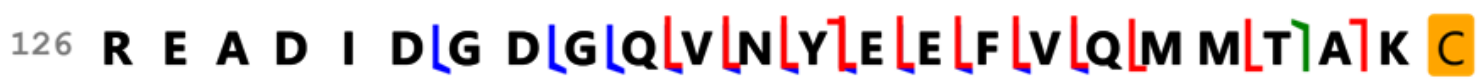

Figure S20: UVPD sequence maps for 7+ charge states of (a) apo-calmodulin with 55\% sequence coverage and (b) $\mathrm{Ca}$ (II) complexes with $43 \%$ sequence coverage. $a / x$ (green), $b / y$ (blue), and $c / z^{*}$ (red) fragment ions are indicated by the color-coded flags. 


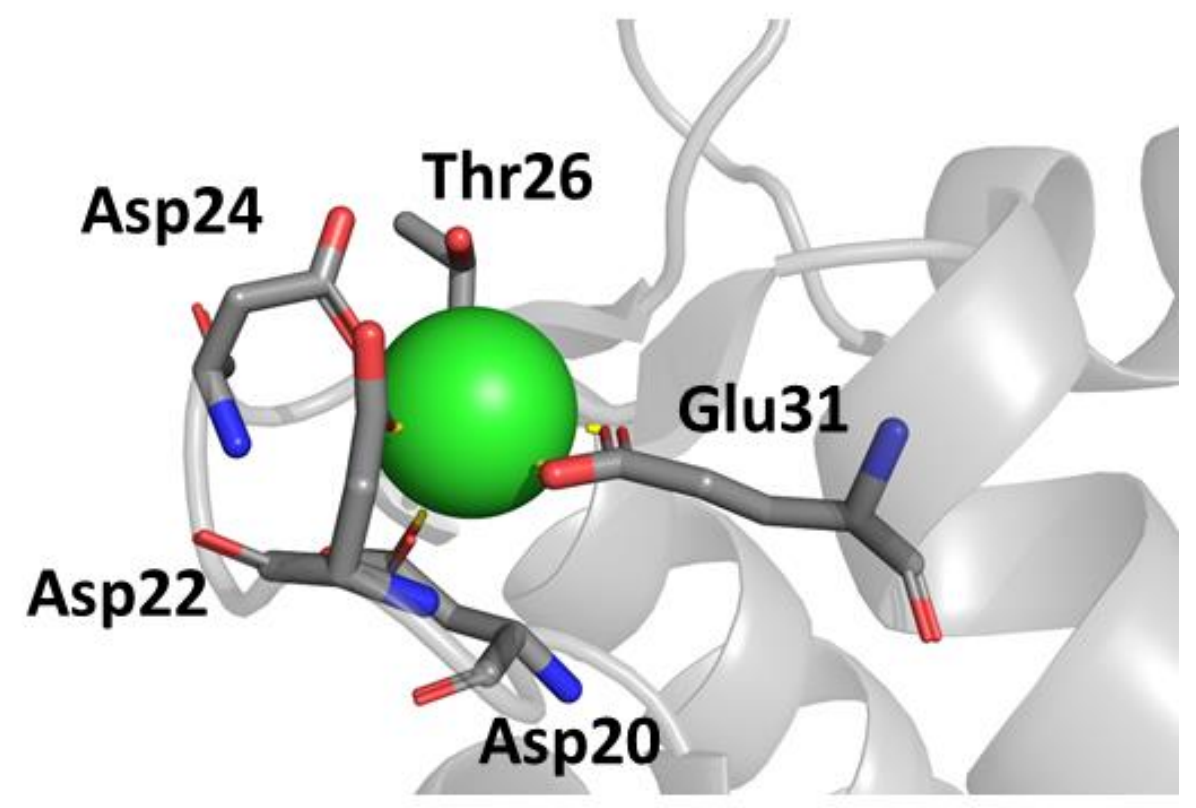

Figure S21: Expansion of the first calcium binding region for calmodulin (PDB: 1CLL), showing the polar contacts (yellow) between the calcium ion (green) and the protein (side-chains). The five residues constituting the binding region for $\mathrm{Ca}(\mathrm{II})$ are Asp20, Asp22, Asp24, Thr26, and Glu31. 


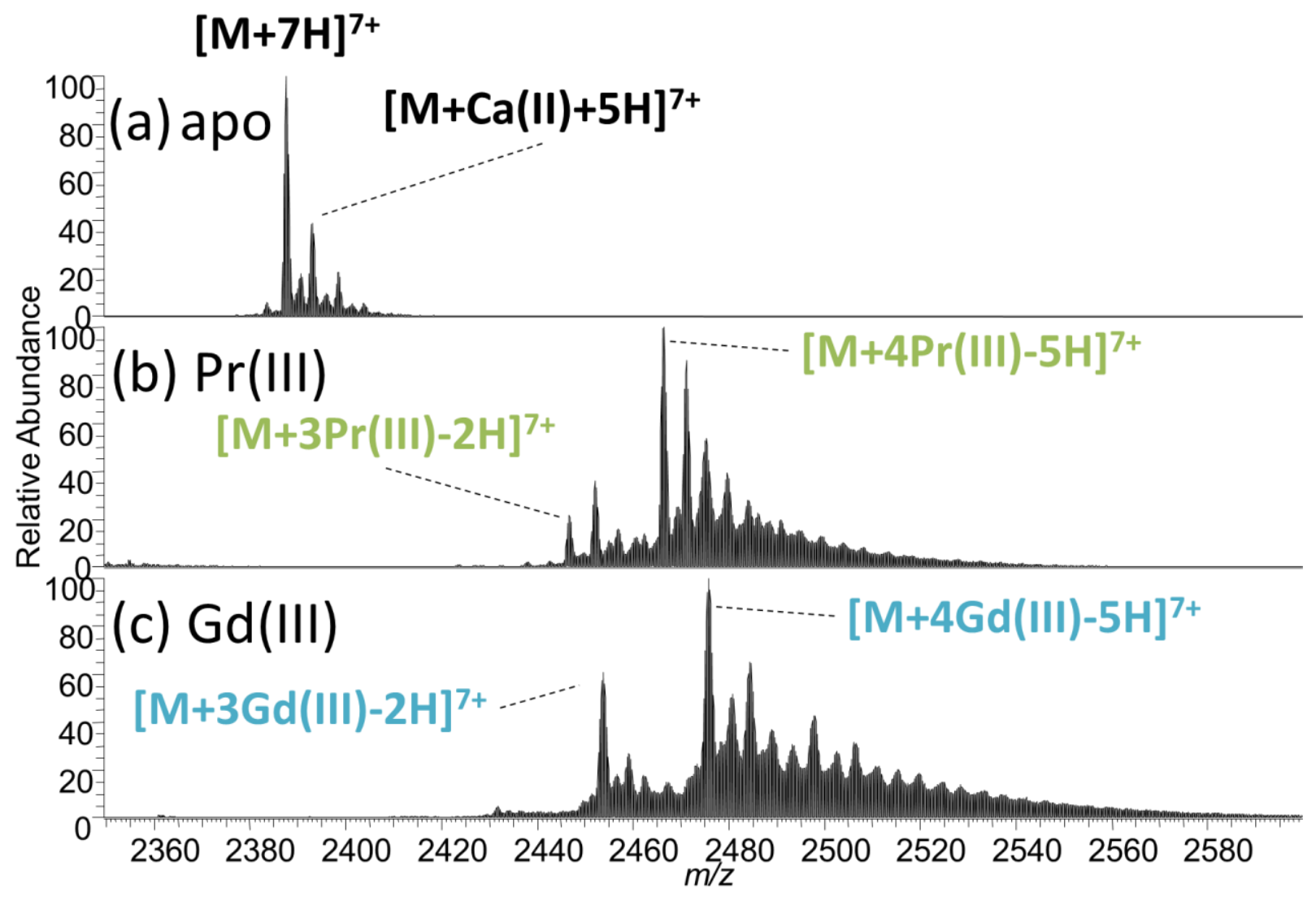

Figure S22: ESI mass spectra of solutions containing calmodulin: (a) no metal, (b) praseodymium acetate, and (c) gadolinium acetate. All solutions contain $120 \mathrm{mM}$ ammonium acetate. 


\section{(a) $\operatorname{Pr}($ III)}
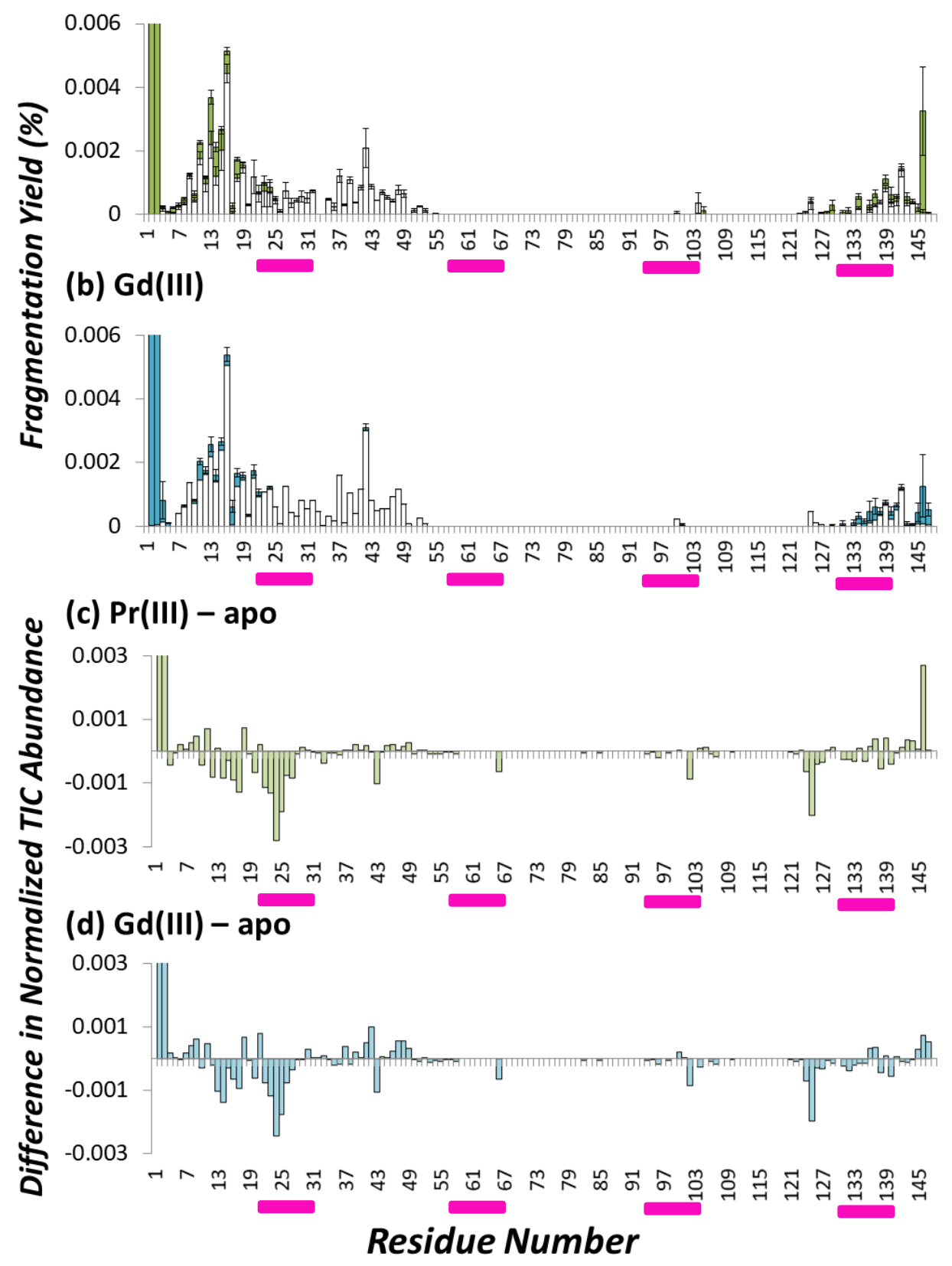

Figure S23: Backbone cleavage yields from UVPD of (a) $\operatorname{Pr}(\mathrm{III})$ and (b) $\mathrm{Gd}(\mathrm{III})$ calmodulin complexes normalized to the TIC. The white bars represent apo fragment ions, and the colorshaded bars represent holo ions, and they are stacked to show the summed apo and holo fragment ions. Difference plot for the UVPD backbone cleavage yields of apo-calmodulin and the (c) $\operatorname{Pr}(\mathrm{III})$ and (d) Gd(III) complexes (7+). Negative values represent regions of the protein backbone for which backbone cleavages of the metal complexes are suppressed relative to the apo-protein. The binding sites for $\operatorname{Pr}(\mathrm{III})$ and $\mathrm{Gd}(\mathrm{III})$ are demarcated in pink bars. 


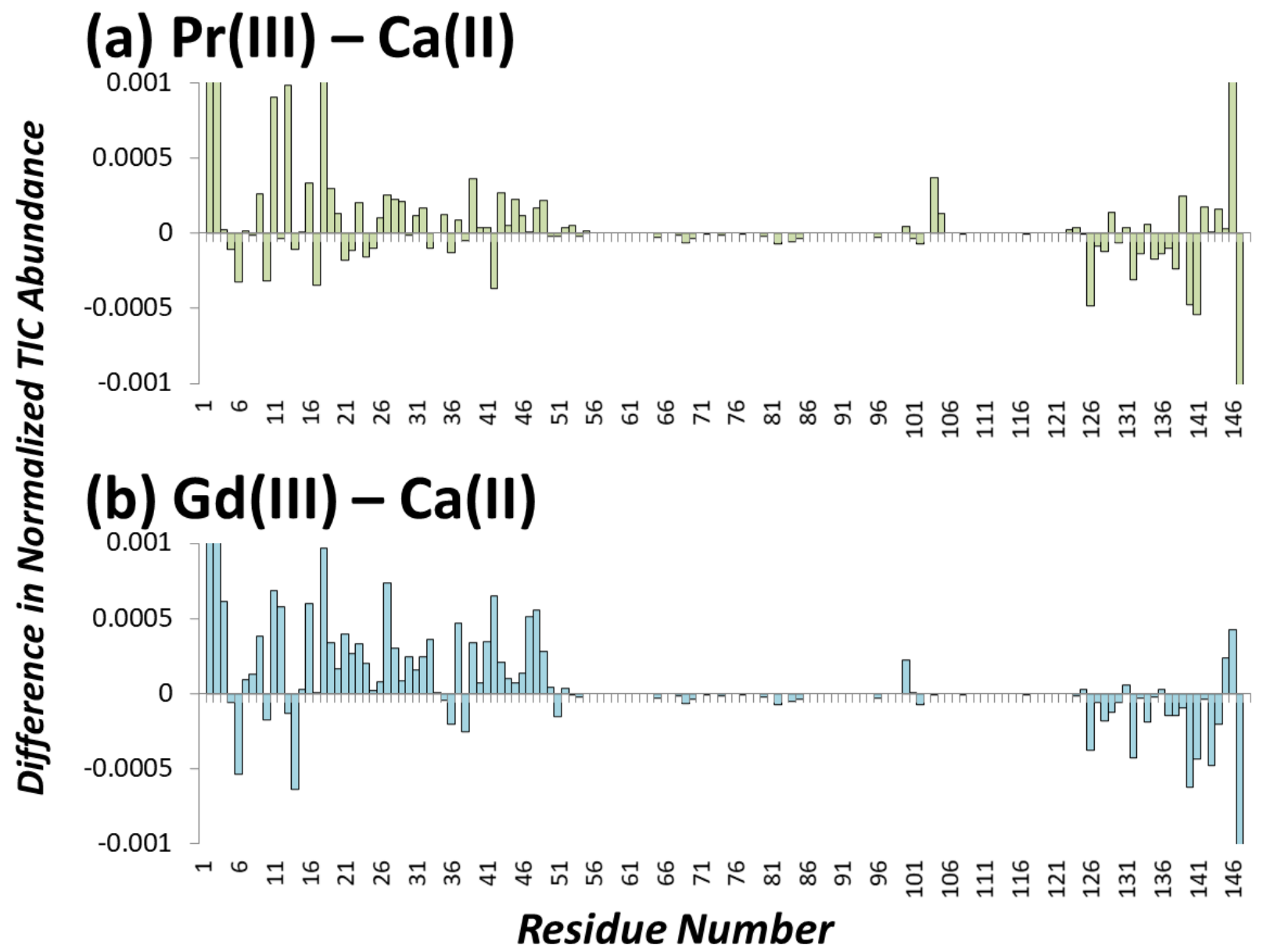

Figure S24: Difference plot for the UVPD backbone cleavage yields of $\mathrm{Ca}(\mathrm{II})$-bound calmodulin and the (a) $\operatorname{Pr}(\mathrm{III})$ and (b) Gd(III) complexes (7+). Negative values represent regions of the protein backbone for which backbone cleavages of the $\mathrm{Ln}$ (III) complexes are suppressed relative to $\mathrm{Ca}$ (II) complexes. 
a
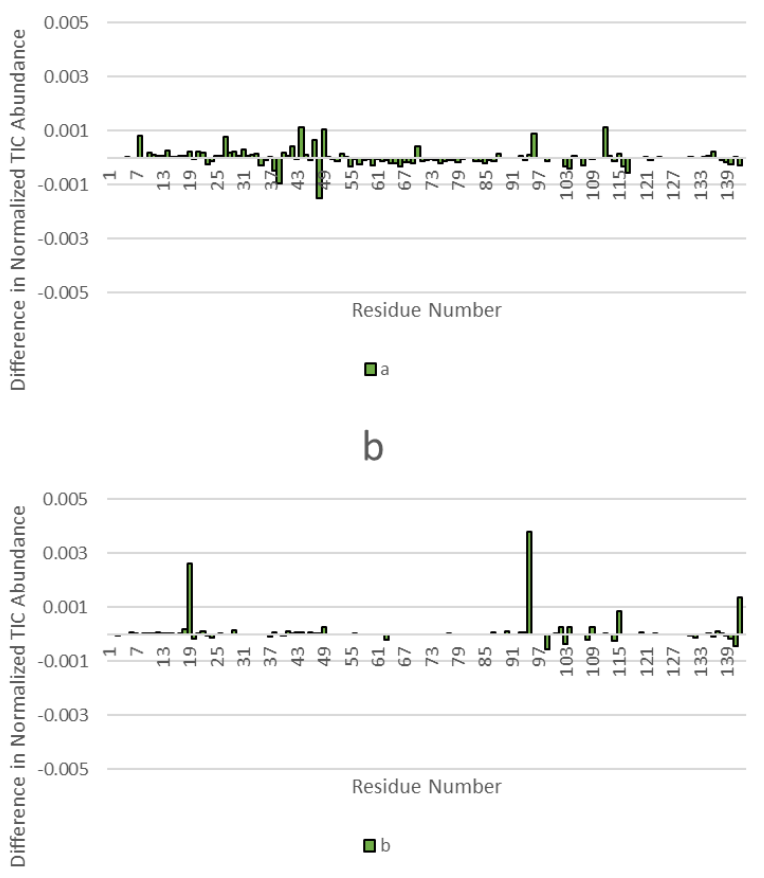

$a+$
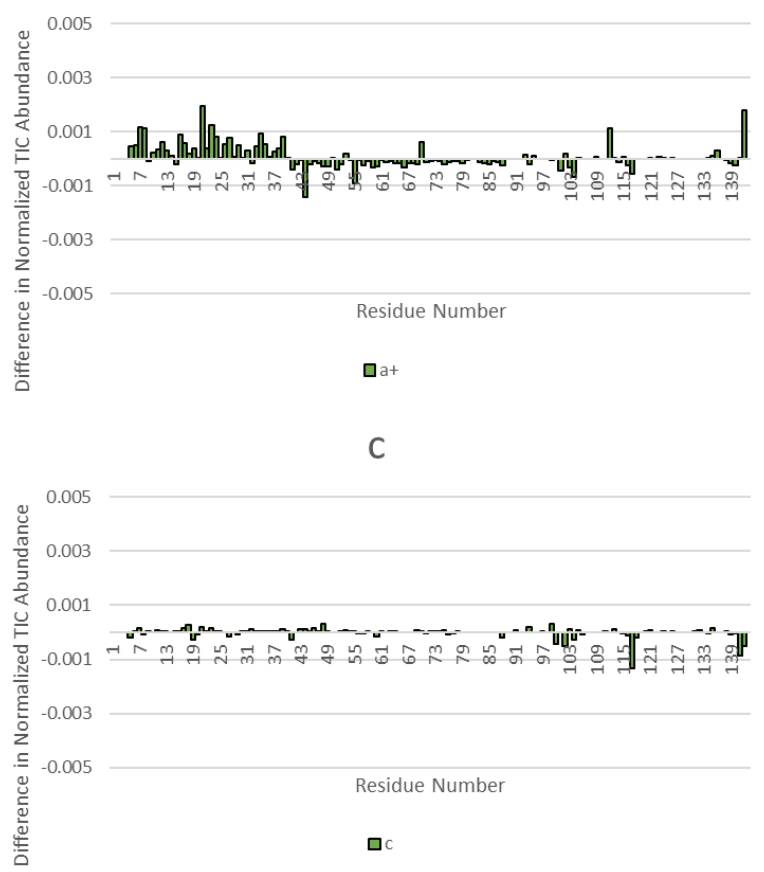

Figure S25. Difference plots showing N-terminal containing ions: SNase $\bullet \operatorname{Pr}^{3+}-$ apo SNase $(\mathrm{a}, \mathrm{a}+1$, b, c) 

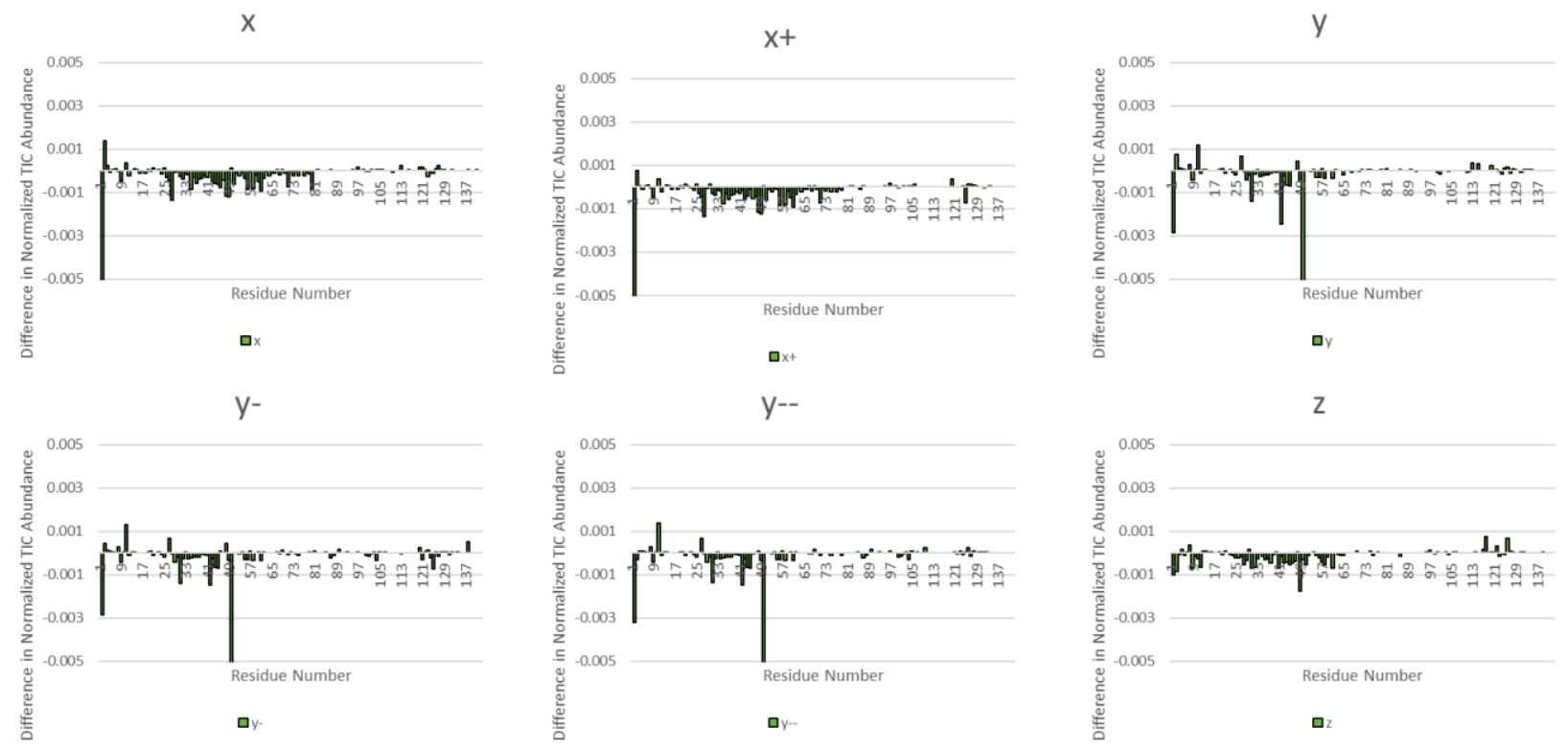

Figure S26. Difference plots showing C-terminal containing ions: SNase $\bullet \mathrm{Pr}^{3+}$ - apo SNase (x, $\mathrm{x}+1, \mathrm{y}, \mathrm{y}-1, \mathrm{y}-2, \mathrm{z})$ 
a

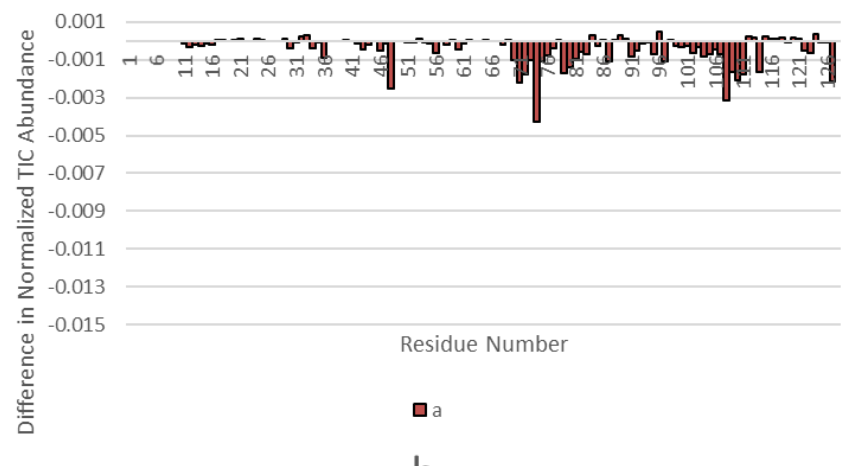

b

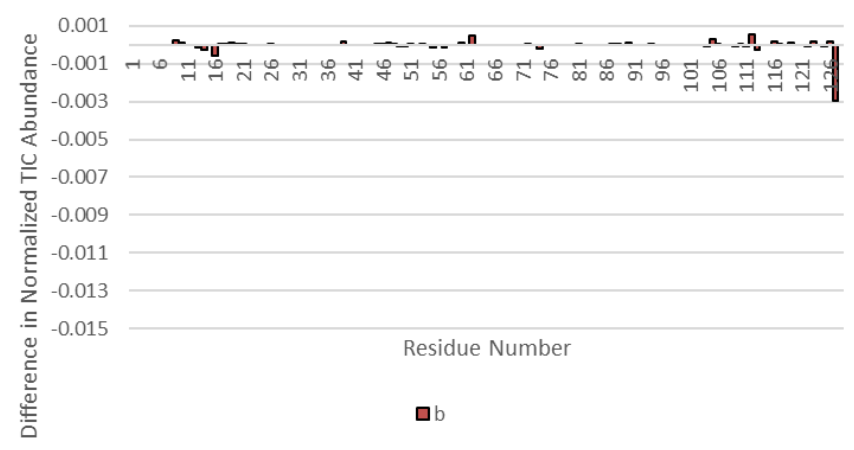

a+

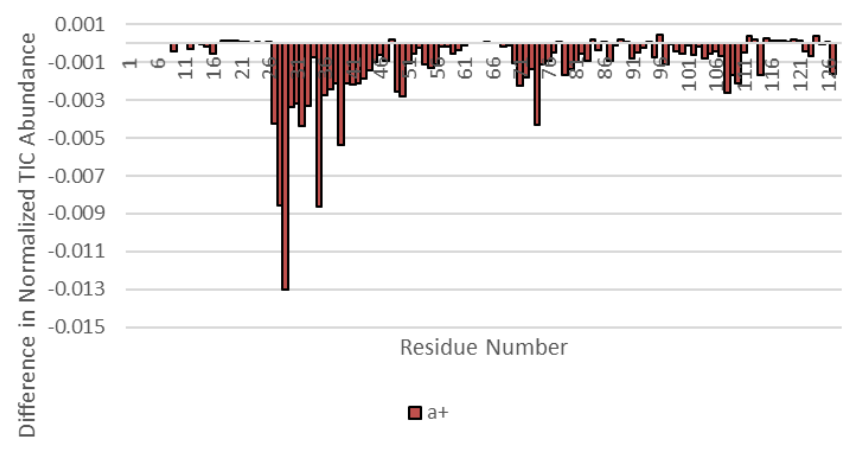

C

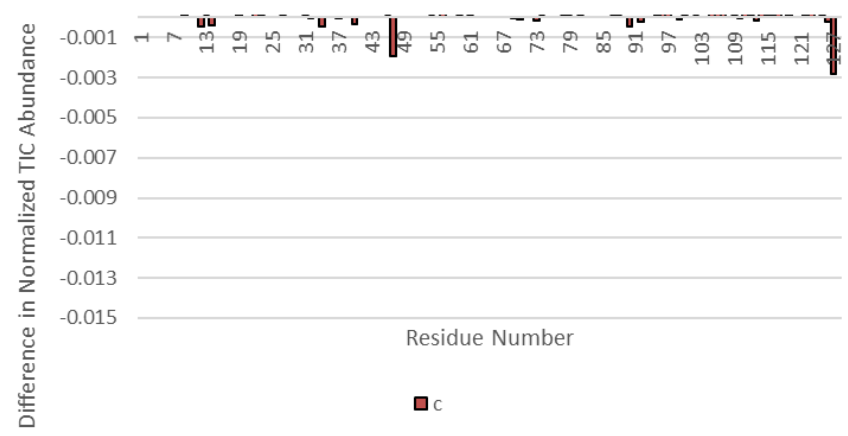

Figure S27. Difference plots showing N-terminal containing ions: Azurin $\cdot \mathrm{Cu}^{2+}-$ apo Azurin (a, $\mathrm{a}+1, \mathrm{~b}, \mathrm{c})$ 

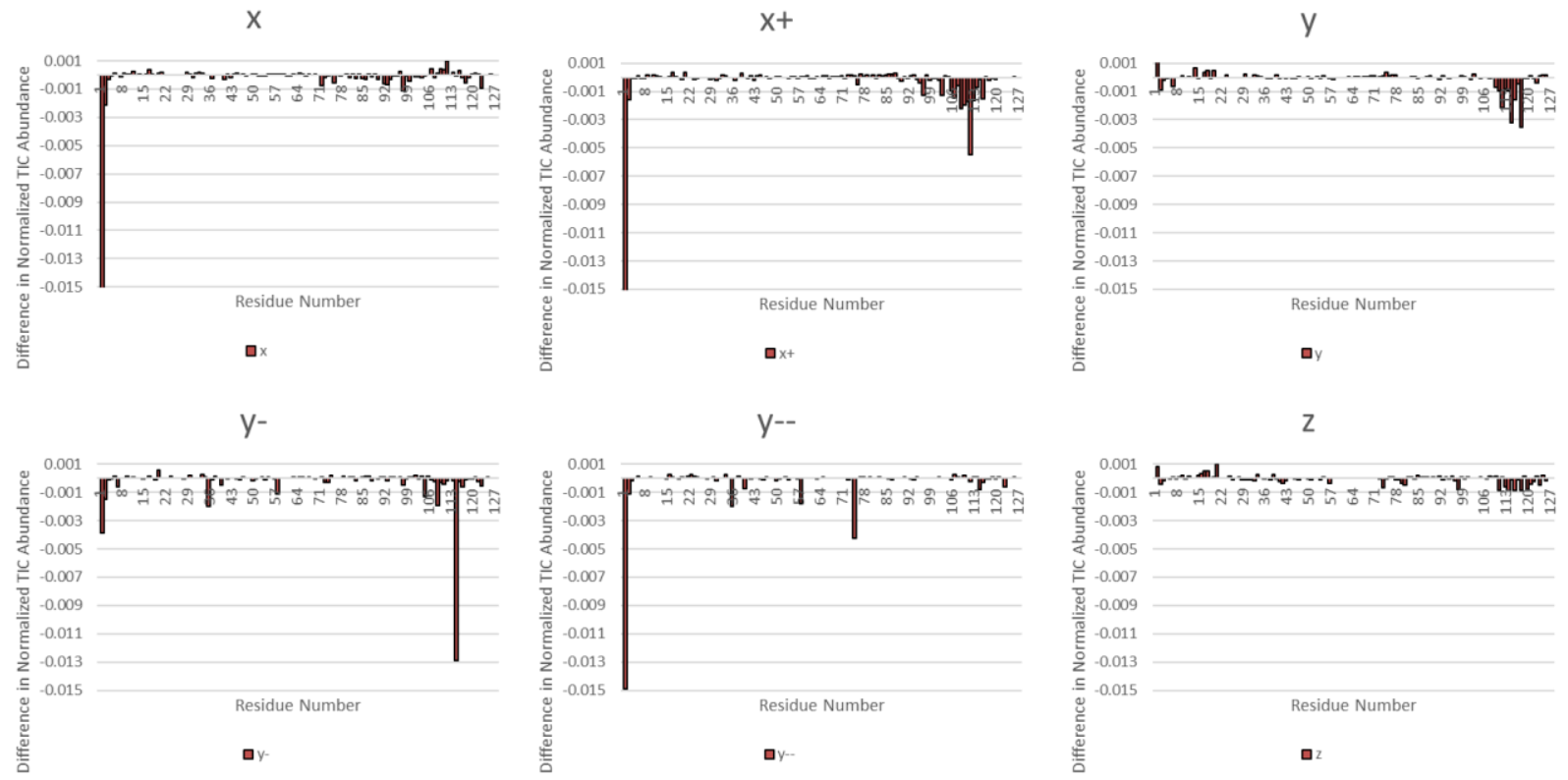

Figure S28. Difference plots showing C-terminal containing ions: Azurin $\bullet \mathrm{Cu}^{2+}-$ apo Azurin $(\mathrm{x}$, $\mathrm{x}+1, \mathrm{y}, \mathrm{y}-1, \mathrm{y}-2, \mathrm{z})$ 
a

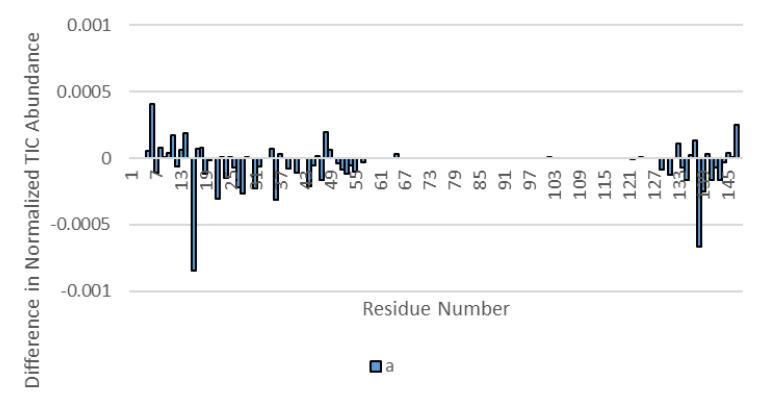

b

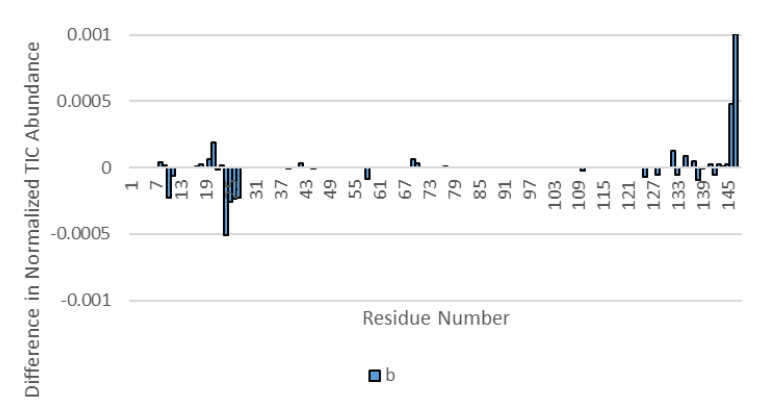

$a+$
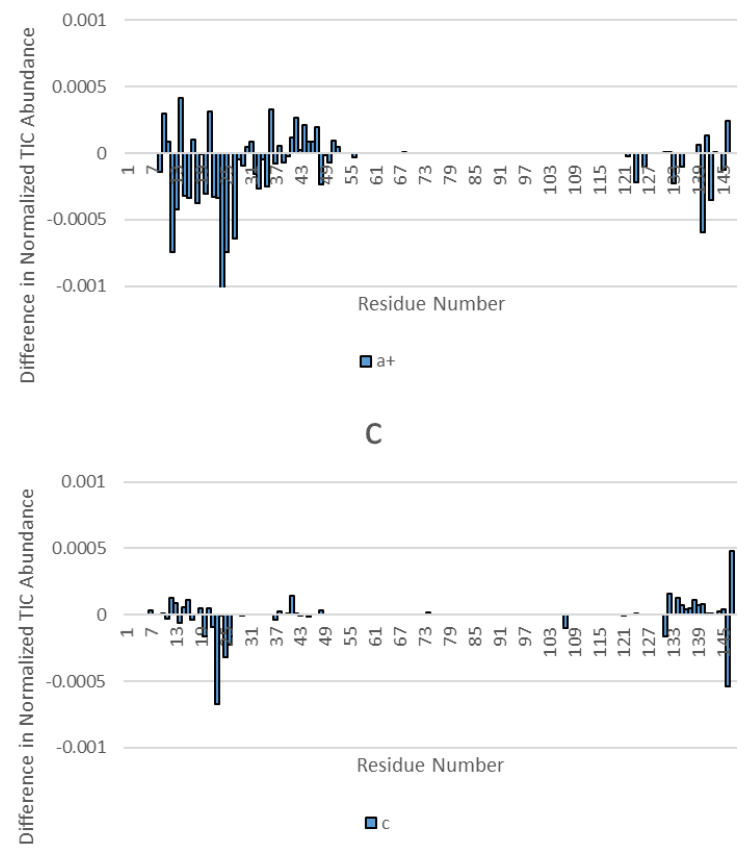

Figure S29. Difference plots showing N-terminal containing ions: Calmodulin $\cdot \mathrm{Ca}^{2+}-$ apo Calmodulin $(a, a+1, b, c)$ 

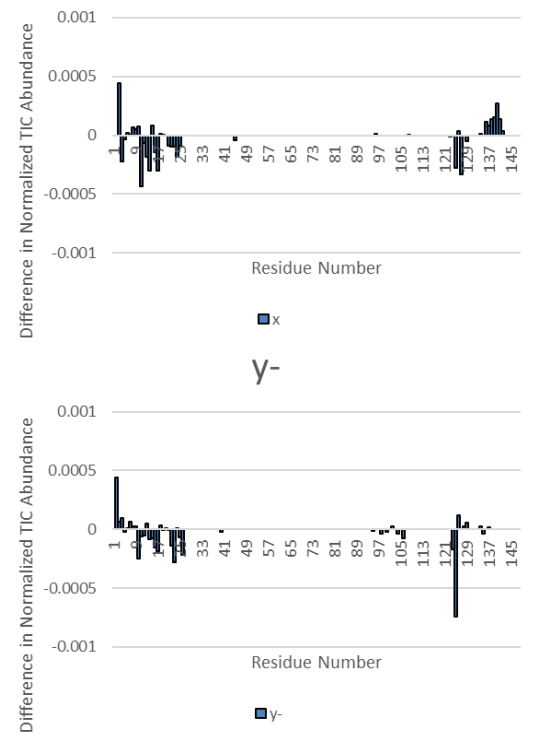

X+
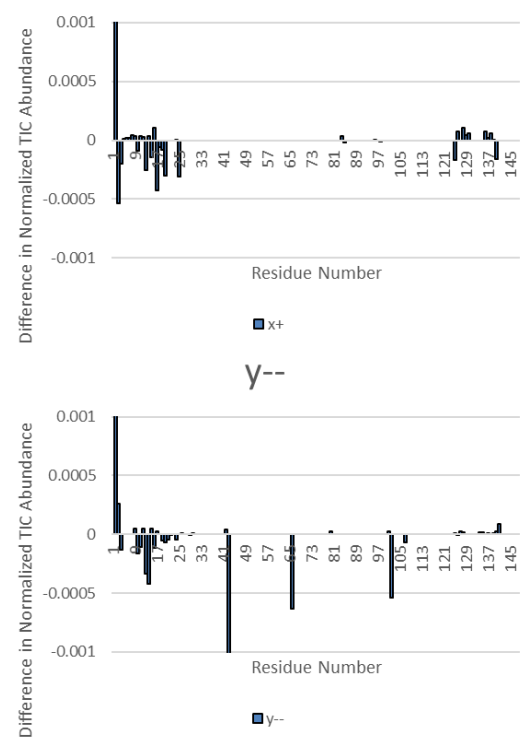

y
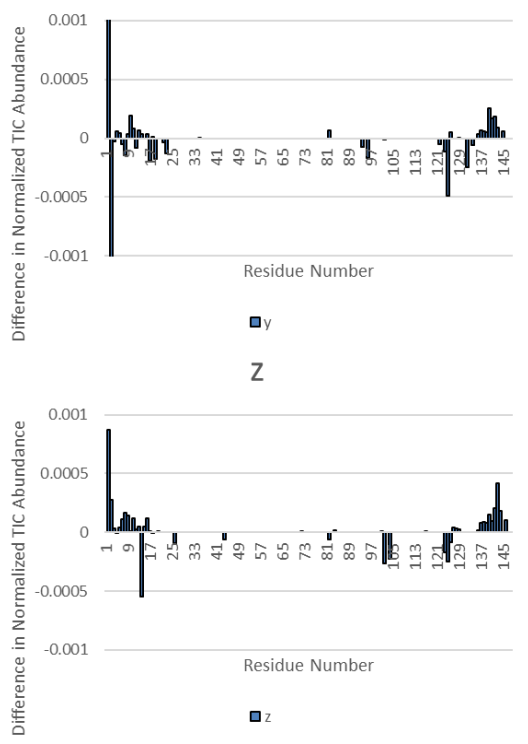

Figure S30. Difference plots showing C-terminal containing ions: Calmodulin $\cdot \mathrm{Ca}^{2+}-$ apo Calmodulin $(\mathrm{x}, \mathrm{x}+1, \mathrm{y}, \mathrm{y}-1, \mathrm{y}-2, \mathrm{z})$ 